\title{
Observability, reconstructibility and state observers of Boolean Control Networks
}

\author{
Ettore Fornasini and Maria Elena Valcher
}

Index Terms-Boolean networks, Boolean control networks, observability, reconstructibility, state observers.

\begin{abstract}
The aim of this paper is to introduce and characterize observability and reconstructibility properties for Boolean networks and Boolean control networks, described according to the algebraic approach proposed by $D$. Cheng and co-authors in the series of papers [3], [6], [7] and in the recent monography [8]. A complete characterization of these properties, based both on the Boolean matrices involved in the network description and on the corresponding digraphs, is provided. Finally, the problem of state observer design for reconstructible $\mathrm{BNs}$ and $\mathrm{BCNs}$ is addressed, and two different solutions are proposed.
\end{abstract}

\section{INTRODUCTION}

Research interests in Boolean networks (BNs) have been motivated by the large number of natural and artificial systems whose describing variables display only two distinct configurations, and hence take only two values. Originally introduced to model simple neural networks, BNs have recently proved to be suitable to describe and simulate the behavior of genetic regulatory networks. Indeed, regulatory genes inside the cells act just like switches, that may take either an "on" or an "off" state (1 and 0, respectively), and this discovery led Kauffman [14] to introduce random Boolean networks as models for genetic networks (see also [23]). As a further application area, $\mathrm{BNs}$ have also been used to describe the interactions among agents and hence to investigate consensus problems [12], [21].

BNs are autonomous systems, since they evolve as automata, whose dynamics is uniquely determined once the initial conditions are assigned. On the other hand, when the network behavior depends also on some (Boolean) control inputs, the concept of BN naturally extends to that of Boolean control network (BCN).

In the last decade, D. Cheng and co-workers have developed an algebraic framework to deal with both BNs and BCNs [3], [4], [5], [6], [7]. Their research efforts resulted in the recent monography [8], where several theoretic problems, ranging from stability and stabilizability to controllability, disturbance decoupling and optimal control, have been investigated. Even more, they stimulated further research in this area (see, for instance [1], [9], [16], [18], [20]), aimed at deepening specific control issues. The main idea underlying this approach is that a Boolean network with $n$ state variables exhibits $2^{n}$ possible configurations, and if any such configuration is represented by means of a canonical vector of size $2^{n}$, all the logic maps

The Authors are with the Dip. di Ingegneria dell'Informazione, Univ. di Padova, via Gradenigo 6/B, 35131 Padova, Italy, phone: +39-049-827-7795 fax: +39-049-827-7614, e-mail:fornasini, meme@dei.unipd.it. that regulate the state-updating can be equivalently described by means of $2^{n} \times 2^{n}$ Boolean matrices. As a result, every Boolean network can be described as a discrete-time linear system. In a similar fashion, a Boolean control network can be converted into a discrete-time bilinear system or, more conveniently, it can be seen as a family of BNs, each of them associated with a specific value of the input variables, and in that sense it represents a switched system. As a consequence of this algebraic set-up, logic-based problems can be converted into algebraic problems and hence solved by resorting to the standard mathematical tools available for linear state-space models and, in particular, for positive state-space models [10], [22], first of all graph theory.

In this paper, by following this stream of research, we first address and characterize observability and reconstructibility of Boolean networks. Then, we extend this analysis to the class of BCNs. Finally, we address the problem of designing a state observer for a $\mathrm{BCN}$. In detail, the paper is organized as follows: in section II we introduce and characterize observability, by first considering two elementary cases (BNs consisting of a single cycle or of a single cycle and some vertices accessing that cycle), and then moving to the general case. Reconstructibility is the focus of section III, where it is proved that this property is equivalent to the observability of the reduced BN consisting of all the states of the BN that belong to some cycle. Observability and reconstructibility for BCNs are introduced and investigated in sections IV and $\mathrm{V}$, respectively. Finally, in section VI, the observer design problem for BCNs (and hence, as a corollary, for BNs), under the reconstructibility assumption, is analyzed, and two different solutions are proposed. A preliminary version of the first part of the paper has been accepted for presentation at the next CDC 2012 conference [11].

Notation. $\mathbb{Z}_{+}$denotes the set of nonnegative integers. Given two integers $k, n \in \mathbb{Z}_{+}$, with $k \leq n$, by the symbol $[k, n]$ we denote the set of integers $\{k, k+1, \ldots, n\}$. We consider Boolean vectors and matrices, taking values in $\mathcal{B}:=\{0,1\}$, with the usual operations (sum + , product $\cdot$ and negation $\urcorner$ ).

$\delta_{k}^{i}$ will denote the $i$ th canonical vector of size $k, \mathcal{L}_{k}$ the set of all $k$-dimensional canonical vectors, and $\mathcal{L}_{k \times n} \subset \mathcal{B}^{k \times n}$ the set of all $k \times n$ matrices whose columns are canonical vectors of size $k$. Any matrix $L \in \mathcal{L}_{k \times n}$ can be represented as a row vector whose entries are canonical vectors in $\mathcal{L}_{k}$, namely $L=\left[\begin{array}{llll}\delta_{k}^{i_{1}} & \delta_{k}^{i_{2}} & \ldots & \delta_{k}^{i_{n}}\end{array}\right]$, for suitable indices $i_{1}, i_{2}, \ldots, i_{n} \in[1, k]$.

A permutation matrix $\Pi$ is a nonsingular square matrix in 
$\mathcal{L}_{k \times k}$. In particular, a matrix

$$
\Pi=C=\left[\begin{array}{ccccc}
0 & 0 & \ldots & 0 & 1 \\
1 & 0 & \ldots & 0 & 0 \\
0 & 1 & \ddots & 0 & 0 \\
\vdots & \vdots & \ddots & \ddots & \vdots \\
0 & 0 & \ldots & 1 & 0
\end{array}\right]=\left[\begin{array}{lllll}
\delta_{k}^{2} & \delta_{k}^{3} & \ldots & \delta_{k}^{k} & \delta_{k}^{1}
\end{array}\right]
$$

is a $k \times k$ cyclic (permutation) matrix.

Given a matrix $L \in \mathcal{B}^{k \times k}$ (in particular, $L \in \mathcal{L}_{k \times k}$ ), we associate with it [2] a digraph $\mathcal{D}(L)$, with vertices $1, \ldots, k$. There is an arc $(j, \ell)$ from $j$ to $\ell$ if and only if the $(\ell, j)$ th entry of $L$ is unitary. A sequence $j_{1} \rightarrow j_{2} \rightarrow \ldots \rightarrow j_{r} \rightarrow$ $j_{r+1}$ in $\mathcal{D}(L)$ is a path of length $r$ from $j_{1}$ to $j_{r+1}$ provided that $\left(j_{1}, j_{2}\right), \ldots,\left(j_{r}, j_{r+1}\right)$ are arcs of $\mathcal{D}(L)$. A closed path is called a cycle. In particular, a cycle $\gamma$ with no repeated vertices is called elementary, and its length $|\gamma|$ coincides with the number of (distinct) vertices appearing in it. Note that a $k \times$ $k$ cyclic matrix has a digraph that consists of one elementary cycle with length $k$.

There is a bijective correspondence between Boolean variables $X \in \mathcal{B}$ and vectors $\mathbf{x} \in \mathcal{L}_{2}$, defined by the relationship

$$
\mathbf{x}=\left[\begin{array}{c}
X \\
\neg X
\end{array}\right]
$$

We introduce the (left) semi-tensor product $\ltimes$ between matrices (and hence, in particular, vectors) as follows [8], [17], [19]: given $L_{1} \in \mathbb{R}^{r_{1} \times c_{1}}$ and $L_{2} \in \mathbb{R}^{r_{2} \times c_{2}}$ (in particular, $L_{1} \in \mathcal{L}_{r_{1} \times c_{1}}$ and $L_{2} \in \mathcal{L}_{r_{2} \times c_{2}}$ ), we set

$L_{1} \ltimes L_{2}:=\left(L_{1} \otimes I_{T / c_{1}}\right)\left(L_{2} \otimes I_{T / r_{2}}\right), \quad T:=$ l.c.m. $\left\{c_{1}, r_{2}\right\}$,

where 1.c.m. denotes the least common multiple. The semitensor product represents an extension of the standard matrix product, by this meaning that if $c_{1}=r_{2}$, then $L_{1} \ltimes L_{2}=L_{1} L_{2}$. Note that if $\mathbf{x}_{1} \in \mathcal{L}_{r_{1}}$ and $\mathbf{x}_{2} \in \mathcal{L}_{r_{2}}$, then $\mathbf{x}_{1} \ltimes \mathbf{x}_{2} \in \mathcal{L}_{r_{1} r_{2}}$. For the various properties of the semi-tensor product we refer to [8]. By resorting to the semi-tensor product, we can extend the previous correspondence to a bijective correspondence between $\mathcal{B}^{n}$ and $\mathcal{L}_{2^{n}}$. This is possible in the following way: given $X=\left[\begin{array}{llll}X_{1} & X_{2} & \ldots & X_{n}\end{array}\right]^{\top} \in \mathcal{B}^{n}$ set

$$
\mathbf{x}:=\left[\begin{array}{c}
X_{1} \\
\neg X_{1}
\end{array}\right] \ltimes\left[\begin{array}{c}
X_{2} \\
\neg X_{2}
\end{array}\right] \ltimes \ldots \ltimes\left[\begin{array}{c}
X_{n} \\
\neg X_{n}
\end{array}\right] .
$$

This amounts to saying that

$$
\mathbf{x}=\left[\begin{array}{c}
X_{1} X_{2} \ldots X_{n-1} X_{n} \\
\left.X_{1} X_{2} \ldots X_{n-1}\right\urcorner X_{n} \\
\left.X_{1} X_{2} \ldots\right\urcorner X_{n-1} X_{n} \\
\vdots \\
\left.\left.\left.\neg X_{1}\right\urcorner X_{2} \ldots\right\urcorner X_{n-1}\right\urcorner X_{n}
\end{array}\right] .
$$

\section{OBSERVABILITy OF BOOLEAN NETWORKS}

A Boolean Network (BN) is described by the following equations

$$
\begin{aligned}
X(t+1) & =f(X(t)), \\
Y(t) & =h(X(t)), \quad t \in \mathbb{Z}_{+},
\end{aligned}
$$

where $X(t)$ and $Y(t)$ denote the $n$-dimensional state variable and the $p$-dimensional output variable at time $t$, taking values in $\mathcal{B}^{n}$ and $\mathcal{B}^{p}$, respectively. $f$ and $h$ are (logic) functions, namely maps $f: \mathcal{B}^{n} \rightarrow \mathcal{B}^{n}$ and $h: \mathcal{B}^{n} \rightarrow \mathcal{B}^{p}$. Upon representing the state and the output vectors $X(t)$ and $Y(t)$ by means of their equivalent $\mathbf{x}(t)$ and $\mathbf{y}(t)$ in $\mathcal{L}_{N}$ and $\mathcal{L}_{P}$, respectively, where $N:=2^{n}$ and $P:=2^{p}$, the $\mathrm{BN}$ (2) can be described [8] as

$$
\begin{aligned}
\mathbf{x}(t+1) & =L \ltimes \mathbf{x}(t)=L \mathbf{x}(t), \\
\mathbf{y}(t) & =H \ltimes \mathbf{x}(t)=H \mathbf{x}(t), \quad t \in \mathbb{Z}_{+},
\end{aligned}
$$

where $L \in \mathcal{L}_{N \times N}$ and $H \in \mathcal{L}_{P \times N}$ are matrices whose columns are canonical vectors of size $N$ and $P$, respectively.

\section{Definition 1: Given a BN (3),}

- two states $\mathbf{x}_{1}=\delta_{N}^{i}$ and $\mathbf{x}_{2}=\delta_{N}^{j}$ are said to be indistinguishable, if the two output evolutions of the $\mathrm{BN}$ starting at $t=0$ from $\mathbf{x}(0)=\mathbf{x}_{1}$ and from $\mathbf{x}(0)=\mathbf{x}_{2}$, respectively ${ }^{1}$, coincide at every time instant $t \in \mathbb{Z}_{+}$; otherwise they are distinguishable;

- the $\mathrm{BN}$ is said to be observable if every two distinct states are distinguishable.

In order to analyze the observability problem, we introduce a family of equivalence relations on the set $\mathcal{L}_{N}$ of all states. We say that $\mathbf{x}_{1}$ and $\mathbf{x}_{2}$ are indistinguishable in $k$ steps $\left(\mathbf{x}_{1} \sim_{k}\right.$ $\mathbf{x}_{2}$ ) if the output evolutions, say $\mathbf{y}_{1}(t)$ and $\mathbf{y}_{2}(t)$, stemming from $\mathbf{x}_{1}(0)=\mathbf{x}_{1}$ and $\mathbf{x}_{2}(0)=\mathbf{x}_{2}$, respectively, coincide for every $t \in[0, k-1]$. The equivalence relation $\sim_{k}$ partitions $\mathcal{L}_{N}$ into disjoint classes. We let $\mathcal{C}_{\sim, k}$ be the set of such classes. It is easily seen that if two states are indistinguishable in $k+1$ steps then they are indistinguishable in $k$ steps, while the converse is not necessarily true. Therefore the cardinality of the set $\mathcal{C}_{\sim, k+1}$ in general is greater than or equal to the cardinality of $\mathcal{C}_{\sim, k}$, and

$$
\left|\mathcal{C}_{\sim, 1}\right| \leq\left|\mathcal{C}_{\sim, 2}\right| \leq\left|\mathcal{C}_{\sim, 3}\right| \leq \ldots
$$

On the other hand, it can be shown [11] that if, for some positive integer $k,\left|\mathcal{C}_{\sim, k}\right|=\left|\mathcal{C}_{\sim, k+1}\right|$, then $\mathcal{C}_{\sim, k}=\mathcal{C}_{\sim, k+1}$ and $\mathcal{C}_{\sim, k}=\mathcal{C}_{\sim, k+\ell}$ for every $\ell \in \mathbb{Z}_{+}$.

Note that condition $\left|\mathcal{C}_{\sim, 1}\right|=1$ corresponds to the situation when all the $\mathrm{BN}$ states produce the same output value, a situation that surely prevents the $\mathrm{BN}$ from being observable. So, when discussing observability, we will always assume that $\rho:=\left|\mathcal{C}_{\sim, 1}\right|$, which coincides with the number of nonzero rows of $H$, is at least 2 . Based on the above reasoning, we can prove a preliminary lemma.

Lemma 1: Given a $\mathrm{BN}$ (3), with $\rho \geq 2$, consider two states $\mathbf{x}_{1}$ and $\mathbf{x}_{2} \in \mathcal{L}_{N}$, and let $\mathbf{y}_{1}(t)$ and $\mathbf{y}_{2}(t), t \in \mathbb{Z}_{+}$, be the output trajectories stemming from $\mathbf{x}_{1}(0)=\mathbf{x}_{1}$ and $\mathbf{x}_{2}(0)=\mathbf{x}_{2}$, respectively. Condition $\mathbf{y}_{1}(t)=\mathbf{y}_{2}(t)$ for every $t \in[0, N-\rho]$ implies $\mathbf{y}_{1}(t)=\mathbf{y}_{2}(t)$ for every $t \in \mathbb{Z}_{+}$.

Proof: We only need to show that the smallest $k$ such that $\left|\mathcal{C}_{\sim, k}\right|=\left|\mathcal{C}_{\sim, k+1}\right|$ cannot be greater than $N-\rho+1$. This follows from the fact that $\left|\mathcal{C}_{\sim, 1}\right|=\rho$ and, therefore, as far

\footnotetext{
${ }^{1}$ In the following, we will denote the state and the output trajectories stemming from $\mathbf{x}_{i}$ by $\mathbf{x}_{i}(t)$ and $\mathbf{y}_{i}(t), t \in \mathbb{Z}_{+}$, respectively. Accordingly, we will use $\mathbf{x}_{i}(0)=\mathbf{x}_{i}$ for the initial state.
} 
as the sequence (4) is strictly increasing we have $\left|\mathcal{C}_{\sim, k}\right| \geq$ $k+\rho-1$. On the other hand, $\left|\mathcal{C}_{\sim, k}\right| \leq N$ for every $k$. This ensures that $\left|\mathcal{C}_{\sim, N-\rho+1}\right|=\left|\mathcal{C}_{\sim, N-\rho+2}\right|$. Therefore, two output trajectories coincide if and only if they coincide on the time interval $[0, N-\rho]$.

As an immediate consequence of the previous lemma, a BN (3) is observable if and only if, given the first $N-\rho+1$ samples of any output trajectory of the BN, $\mathbf{y}(t), t \in[0, N-\rho]$, we can determine the initial condition $\mathbf{x}(0)$ that has generated it. In fact, (see Example 1, below), this bound is tight, by this meaning that there exist BNs for which $N-\rho+1$ output values are required to determine the initial state. Lemma 1 immediately leads to the following condition, which reminds of the analogous one obtained for linear systems.

Proposition 1: A BN (3) is observable if and only if the observability matrix in $N-1$ steps

$$
\mathcal{O}_{N-1}:=\left[\begin{array}{c}
H \\
H L \\
H L^{2} \\
\vdots \\
H L^{N-2}
\end{array}\right]
$$

has $N$ distinct columns.

As a further step, we want to relate the observability of a $\mathrm{BN}$ (3) to the structure of the associated digraph $\mathcal{D}(L)$. To this goal, we first consider a BN whose digraph $\mathcal{D}(L)$ contains a single cycle and all the other states access the cycle.

Proposition 2: Consider a BN (3), with

$$
L=\left[\begin{array}{cc}
W & 0 \\
T & C
\end{array}\right] \in \mathcal{L}_{N \times N},
$$

where $C$ is a $k \times k$ cyclic matrix and $W$ is nilpotent. The BN is observable if and only if

i) [distinguishability of states before state merging] if $i \neq j$, condition $L \delta_{N}^{i}=L \delta_{N}^{j}$ implies $H \delta_{N}^{i} \neq H \delta_{N}^{j}$;

ii) [distinguishability of states belonging to the cycle] every state belonging to the cycle, $\delta_{N}^{i}, i \in[N-k+1, N]$, generates a periodic output trajectory with minimal period $k$.

Proof: [Necessity] If condition i) was not satisfied, the two initial states $\mathbf{x}_{1}(0)=\delta_{N}^{i}$ and $\mathbf{x}_{2}(0)=\delta_{N}^{j}$ would produce the same state trajectory, starting at $t=1$, and the corresponding output trajectories, $\mathbf{y}_{1}(t)$ and $\mathbf{y}_{2}(t), t \in \mathbb{Z}_{+}$, would coincide for every $t \in \mathbb{Z}_{+}$. Hence the two states would be indistinguishable. On the other hand, if condition ii) is not satisfied, there would be a state belonging to the cycle, say $\mathbf{x}(0)=\delta_{N}^{i}, i \in[N-k+1, N]$, generating a periodic output trajectory with minimal period $\bar{k}$, a proper divisor of $k$, and hence it would be $\mathbf{y}(t+\bar{k})=\mathbf{y}(t)$ for every $t \in \mathbb{Z}_{+}$. Consequently, the two states $\mathbf{x}(0) \neq \mathbf{x}(\bar{k})$, belonging to the cycle, would be indistinguishable.

[Sufficiency] We want to prove that if conditions i) and ii) hold, then the BN is observable. Suppose it is not. Then two distinct states $\mathbf{x}_{1}(0)=\mathbf{x}_{1}$ and $\mathbf{x}_{2}(0)=\mathbf{x}_{2}$ can be found that produce the same output trajectories, i.e. $\mathbf{y}_{1}(t)=\mathbf{y}_{2}(t), \forall t \in$
$\mathbb{Z}_{+}$. If the two state trajectories eventually coincide, then there exists a minimum $t^{\prime} \in \mathbb{Z}_{+}$such that $\mathbf{x}_{1}\left(t^{\prime}+1\right)=\mathbf{x}_{2}\left(t^{\prime}+1\right)$. But then assumption i) is contradicted for the two distinct states $\mathbf{x}_{1}\left(t^{\prime}\right)=\delta_{N}^{i}$ and $\mathbf{x}_{2}\left(t^{\prime}\right)=\delta_{N}^{j}$. So, we now assume that, at every time $t \in \mathbb{Z}_{+}, \mathbf{x}_{1}(t) \neq \mathbf{x}_{2}(t)$. Consider the sequence of pairs $\left(\mathbf{x}_{1}(t), \mathbf{x}_{2}(t)\right), t \in \mathbb{Z}_{+}$. Since all such pairs take values in the finite set $\mathcal{L}_{N} \times \mathcal{L}_{N}$, there exist $t_{m}, t_{M} \in \mathbb{Z}_{+}$, with $t_{m}<$ $t_{M}$, such that $\left(\mathbf{x}_{1}\left(t_{m}\right), \mathbf{x}_{2}\left(t_{m}\right)\right)=\left(\mathbf{x}_{1}\left(t_{M}\right), \mathbf{x}_{2}\left(t_{M}\right)\right)$. As both the trajectories $\mathbf{x}_{1}(t)$ and $\mathbf{x}_{2}(t)$ are periodic starting (at least) from $t=t_{m}$, this means that $\mathbf{x}_{1}\left(t_{m}\right)=\delta_{N}^{i}$ and $\mathbf{x}_{2}\left(t_{m}\right)=$ $\delta_{N}^{j}, i \neq j$, are indistinguishable states corresponding to the cyclic part $C$. So, both these states, belonging to the cycle, generate a periodic output trajectory whose minimal period is smaller than $k$.

Remark 1: Condition ii) in Proposition 2 can be simply restated by saying that the ordered $k$-tuple $(\mathbf{y}(0), \mathbf{y}(1), \ldots, \mathbf{y}(k-1))$ corresponding to any periodic state trajectory is irreducible, namely it cannot be obtained by repeating a shorter ordered sequence.

Example 1: Consider the BN (3) with $L=C$ an $N \times N$ cyclic matrix and

$$
H=\left[\begin{array}{lllll}
\delta_{2}^{1} & \delta_{2}^{1} & \ldots & \delta_{2}^{1} & \delta_{2}^{2}
\end{array}\right] \in \mathcal{L}_{2 \times N} .
$$

We may describe the BN by means of a suitable digraph, obtained by adding to $\mathcal{D}(L)$ the information regarding the static output map $H$. This can be achieved by associating with each node of $\mathcal{D}(L)$ a dashed arrow, labeled by the value of the corresponding output.

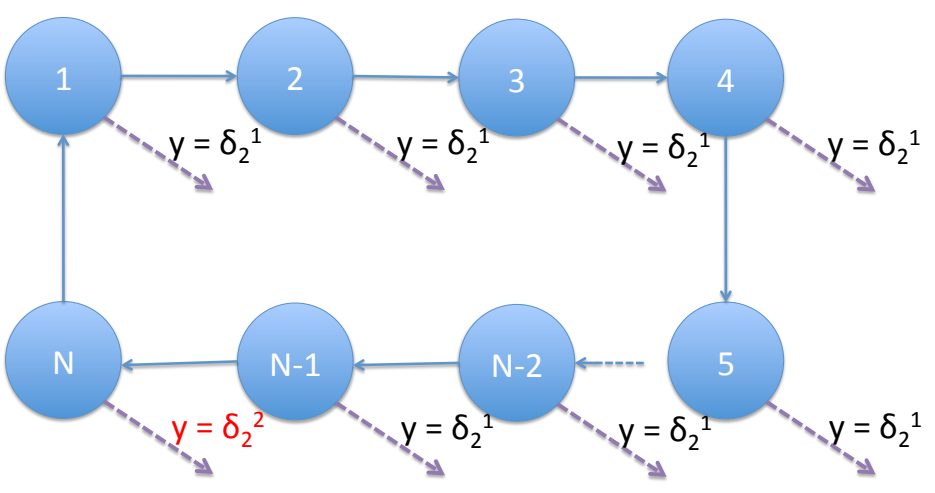

FIG. 1. Digraph corresponding to the BN of Example 1.

By Proposition 2 , the $\mathrm{BN}$ is observable. In order to determine $\mathbf{x}(0)$, consider the sequence $\mathbf{y}(0), \mathbf{y}(1), \ldots, \mathbf{y}(N-2)$. If all such vectors are equal to $\delta_{2}^{1}$, then $\mathbf{x}(0)=\delta_{N}^{1}$, otherwise if $\mathbf{y}(t)=\delta_{2}^{2}$ for some $t \in[0, N-2]$, then $\mathbf{x}(t)=\delta_{N}^{N}$ and hence $\mathbf{x}(0)=\delta_{N}^{N-t}$. Note that, in general, it would not be possible to determine the initial state by stopping the output observation before time $t=N-2$. Finally, the observability matrix in $N-1$ steps is 
$\mathcal{O}_{N-1}=\left[\begin{array}{c}H \\ H L \\ H L^{2} \\ \vdots \\ H L^{N-2}\end{array}\right]=\left[\begin{array}{cccccc}\delta_{2}^{1} & \delta_{2}^{1} & \ldots & \delta_{2}^{1} & \delta_{2}^{1} & \delta_{2}^{2} \\ \delta_{2}^{1} & \delta_{2}^{1} & \ldots & \delta_{2}^{1} & \delta_{2}^{2} & \delta_{2}^{1} \\ \delta_{2}^{1} & \delta_{2}^{1} & \ldots & \delta_{2}^{2} & \delta_{2}^{1} & \delta_{2}^{1} \\ \vdots & \vdots & . & \vdots & \vdots & \vdots \\ \delta_{2}^{1} & \delta_{2}^{2} & \ldots & \delta_{2}^{1} & \delta_{2}^{1} & \delta_{2}^{1}\end{array}\right]$

Proposition 3: Consider a BN (3), with

$$
\begin{aligned}
L & =\operatorname{blockdiag}\left\{D_{1}, D_{2}, \ldots, D_{r}\right\} \in \mathcal{L}_{N \times N} \\
\text { and } \quad D_{\nu} & =\left[\begin{array}{cc}
W_{\nu} & 0 \\
T_{\nu} & C_{\nu}
\end{array}\right] \in \mathcal{L}_{n_{\nu} \times n_{\nu}},
\end{aligned}
$$

where $W_{\nu}$ is a $\left(n_{\nu}-k_{\nu}\right) \times\left(n_{\nu}-k_{\nu}\right)$ nilpotent matrix, and $C_{\nu}$ is a $k_{\nu} \times k_{\nu}$ cyclic matrix. The $\mathrm{BN}$ is observable if and only if

i) [distinguishability of states before state merging] if $i \neq j$, condition $L \delta_{N}^{i}=L \delta_{N}^{j}$ implies $H \delta_{N}^{i} \neq H \delta_{N}^{j}$;

ii) [distinguishability of states belonging to a cycle] for every $\ell \in[1, r]$, the ordered $k_{\ell}$-tuple $\left(H \delta_{N}^{i+1}, H \delta_{N}^{i+2}\right.$, $\left.\ldots, H \delta_{N}^{i+k_{\ell}}\right)$, with $^{2} i:=\left(n_{1}+n_{2}+\ldots+n_{\ell-1}\right)+\left(n_{\ell}-k_{\ell}\right)$, is irreducible;

iii) [distinguishability of states belonging to different cycles] if $\ell, d \in[1, r], \ell \neq d$, and $k_{\ell}=k_{d}=: k$, the two ordered $k$-tuples

$$
\left(H \delta_{N}^{i+1}, H \delta_{N}^{i+2}, \ldots, H \delta_{N}^{i+k}\right), \quad\left(H \delta_{N}^{j+1}, H \delta_{N}^{j+2}, .\right.
$$

with $i=\left(n_{1}+n_{2}+\ldots+n_{\ell-1}\right)+\left(n_{\ell}-k_{\ell}\right)$ and $j=$ $\left(n_{1}+n_{2}+\ldots+n_{d-1}\right)+\left(n_{d}-k_{d}\right)$, neither coincide nor can be obtained one from the other by means of cyclic permutations.

Proof: [Necessity] The necessity of conditions i) and ii) follows immediately from Proposition 2. On the other hand, if condition iii) was not satisfied, there would be two initial states corresponding to two distinct cycles and generating the same periodic output trajectory, thus contradicting observability.

[Sufficiency] We want to prove that if conditions i), ii) and iii) hold, then the BN is observable. Suppose it is not. Then two distinct states $\mathbf{x}_{1}(0)=\mathbf{x}_{1}$ and $\mathbf{x}_{2}(0)=\mathbf{x}_{2}$ could be found that produce the same output trajectory. If $\mathbf{x}_{1}$ and $\mathbf{x}_{2}$ correspond to the same block $D_{\nu}$ then, by the same reasoning adopted in the proof of Proposition 2, either i) or ii) would be contradicted. So, assume that such indistinguishable states correspond to different blocks, say $D_{\ell}$ and $D_{d}, \ell \neq d$. Since both state trajectories eventually become periodic of periods $k_{\ell}$ and $k_{d}$, respectively, the corresponding output trajectories become periodic, too, and since condition ii) holds, the minimal periods of the outputs coincide with the minimal periods of the state trajectories. Since the output trajectories coincide, by the indistinguishability of the states, it follows that $k_{\ell}=k_{d}$. Set $k:=k_{\ell}=k_{d}$ and let $t_{m} \in \mathbb{Z}_{+}$be the smallest time $t$ such that $\mathbf{x}_{1}(t)=\delta_{N}^{i+1}$ for $i=\left(n_{1}+n_{2}+\ldots+\right.$ $\left.n_{\ell-1}\right)+\left(n_{\ell}-k_{\ell}\right)$, and assume that $\mathbf{x}_{2}\left(t_{m}\right)=\delta_{N}^{h+1}$ for some

\footnotetext{
${ }^{2}$ We assume $n_{0}:=0$.
}

$h \in\left[\left(n_{1}+n_{2}+\ldots+n_{d-1}\right)+\left(n_{d}-k_{d}\right), n_{1}+n_{2}+\ldots+n_{d}-1\right]$. Then the two sequences

$$
\left(H \delta_{N}^{i+1}, H L \delta_{N}^{i+1}, \ldots, H L^{k-1} \delta_{N}^{i+1}\right),
$$$$
\left(H \delta_{N}^{h+1}, H L \delta_{N}^{h+1}, \ldots, H L^{k-1} \delta\right.
$$

· coincide, thus contradicting iii).

Remark 2: Conditions i), ii) and iii) of Proposition 3 can be expressed in a rather compact form if we block-partition the matrix $H$, according to the block partition of $L$. Indeed, if we assume that

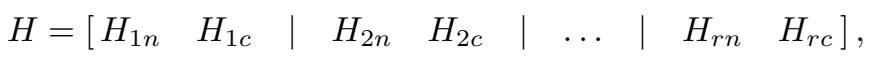

where each block $\left[\begin{array}{ll}H_{\nu n} & H_{\nu c}\end{array}\right]$ has size $P \times n_{\nu}$, while $H_{\nu c}$ has size $P \times k_{\nu}$, then the $\mathrm{BN}$ (3) is observable if and only if i) each block $\left[\begin{array}{cc}W_{\nu} & 0 \\ T_{\nu} & C_{\nu} \\ H_{\nu n} & H_{\nu c}\end{array}\right]$ has all distinct columns;

ii) each block $H_{\nu c}$ cannot be seen as the juxtaposition of two or more copies of the same block (say $\Phi_{\nu}$ ), i.e. $H_{\nu c} \neq$ $\left[\begin{array}{llll}\Phi_{\nu} & \Phi_{\nu} & \ldots & \Phi_{\nu}\end{array}\right]$

iii) if $\ell \neq d$, the blocks $H_{\ell c}$ and $H_{d c}$ are distinct and cannot be obtained one from the other by means of cyclic permutations of the columns, i.e. $\nexists$ a cyclic matrix $C$ and $h \in \mathbb{Z}_{+}$such that $H_{d c}=H_{\ell c} C^{h}$.

Proposition 3 provides a general characterization of observ-


matrix $L$ of every $\mathrm{BN}$ can be reduced to the block diagonal form (6)-(7), by means of a suitable permutation matrix. Indeed, every state trajectory of a BN takes only a finite number of distinct values and hence it eventually becomes periodic (possibly constant). The fact that all columns of $L$ are canonical vectors implies that the set of all states of the BN can be partitioned into say $r$ (disjoint) domains of attraction, each of them consisting of an elementary cycle (called equilibrium point, in case it consists of a single state) and a number of states that eventually converge to it.

Proposition 4: Given a BN (3), there exists $r \in \mathbb{N}$ and a permutation matrix $\Pi$ such that

$$
\begin{aligned}
\Pi^{\top} L \Pi & =\operatorname{blockdiag}\left\{D_{1}, D_{2}, \ldots, D_{r}\right\} \in \mathcal{L}_{N \times N}(8) \\
\text { with } \quad D_{\nu} & =\left[\begin{array}{cc}
W_{\nu} & 0 \\
T_{\nu} & C_{\nu}
\end{array}\right] \in \mathcal{L}_{n_{\nu} \times n_{\nu}},
\end{aligned}
$$

where $W_{\nu}$ is a $\left(n_{\nu}-k_{\nu}\right) \times\left(n_{\nu}-k_{\nu}\right)$ nilpotent matrix, and $C_{\nu}$ is a $k_{\nu} \times k_{\nu}$ cyclic matrix.

Proof: Let $\mathcal{Z}_{1}, \mathcal{Z}_{2}, \ldots, \mathcal{Z}_{r}$ be the distinct elementary cycles (possibly equilibrium points) of the system, and let $\mathcal{D}_{1}, \mathcal{D}_{2}, \ldots, \mathcal{D}_{r}$ be the corresponding disjoint domains of attraction. Clearly, a permutation matrix $\Pi$ can be found such that $\Pi^{\top} L \Pi$ is block diagonal as in (8). Even more, we can order the states of each domain $\mathcal{D}_{\nu}$ so that those belonging to $\mathcal{Z}_{\nu}$ are the last ones. So, it entails no loss of generality assuming that each diagonal block is as in (9), where $C_{\nu}$ is the cyclic matrix associated with $\mathcal{Z}_{\nu}$. All the states of $\mathcal{D}_{\nu} \backslash \mathcal{Z}_{\nu}$ 
produce trajectories that belong to $\mathcal{Z}_{\nu}$ after a finite number of steps, which means that, for a sufficiently high $k$,

$$
D_{\nu}^{k}=\left[\begin{array}{cc}
W_{\nu}^{k} & 0 \\
* & C_{\nu}^{k}
\end{array}\right]=\left[\begin{array}{cc}
0 & 0 \\
* & C_{\nu}^{k}
\end{array}\right],
$$

and hence $W_{\nu}$ is nilpotent.

Propositions 3 and 4 have mainly a theoretical value, as they connect the topological structure of the digraph $\mathcal{D}(L)$ to the observability property. However, the evaluation of the permutation matrix $\Pi$ is computationally demanding, as it requires to determine the limit cycles and the corresponding domains of attraction. As a matter of fact, observability of a BN can be characterized in a much simpler way, whose practical feasibility will be addressed in Remark 4. To this goal it is sufficient to notice that every elementary cycle of length $k$ corresponds to $k$ distinct periodic state trajectories, depending on the specific choice of the initial state. On the other hand, each of these $k$ periodic trajectories is uniquely associated with the ordered $k$-tuple $(\mathbf{x}(0), \mathbf{x}(1), \ldots, \mathbf{x}(k-1))$. By taking this perspective, we can merge the distinguishability conditions ii) and iii) into a single one.

Theorem 1: A BN (3) is observable if and only if

i) [distinguishability of states before state merging] for every $\mathbf{x}_{1}, \mathbf{x}_{2} \in \mathcal{L}_{N}$, with $\mathbf{x}_{1} \neq \mathbf{x}_{2}$, condition $L \mathbf{x}_{1}=L \mathbf{x}_{2}$ implies $H \mathbf{x}_{1} \neq H \mathbf{x}_{2}$;

ii) [distinguishability of states belonging to (the same or different) cycles] for every pair of distinct periodic state trajectories of the same minimal period $k$, described by the two ordered $k$-tuples

$$
\left(\mathbf{x}_{1}, \mathbf{x}_{2}, \ldots, \mathbf{x}_{k}\right) \neq\left(\overline{\mathbf{x}}_{1}, \overline{\mathbf{x}}_{2}, \ldots, \overline{\mathbf{x}}_{k}\right),
$$

the corresponding output trajectories are periodic with (minimal) period $k$ and described by two different ordered $k$-tuples, i.e.

$$
\left(H \mathbf{x}_{1}, H \mathbf{x}_{2}, \ldots, H \mathbf{x}_{k}\right) \neq\left(H \overline{\mathbf{x}}_{1}, H \overline{\mathbf{x}}_{2}, \ldots, H \overline{\mathbf{x}}_{k}\right) .
$$

\section{RECONSTRUCTIBILITY OF BOOLEAN NETWORKS}

In the previous section we have seen that, as for linear statespace models, observability corresponds to the possibility of uniquely determining the system initial condition $\mathbf{x}(0)$ from the observation of the corresponding output evolution in some interval $[0, T]$. Reconstructibility property may be introduced along the same perspective, as the possibility of determining the system final state $\mathbf{x}(T)$ from the corresponding output evolution in $[0, T]$.

Definition 2: A BN (3) is said to be reconstructible if there exists $T \in \mathbb{Z}_{+}$such that the knowledge of the output trajectory $\mathbf{y}(t), t \in[0, T]$, allows to uniquely determine $\mathbf{x}(T)$ (and hence $\mathbf{x}(t)$ for every $t \geq T)$. If this is the case, the smallest such $T$ will be denoted by $T_{\min }$.

It is clear that observability implies reconstructibility. On the other hand, it is also obvious that if all the states of a $\mathrm{BN}$ belong to some cycle (or, in particular, are equilibrium points), then once the state $\mathbf{x}(T)$ has been uniquely identified, the state $\mathbf{x}(0)$ can be determined by moving backward. This means that, for a $\mathrm{BN}$ whose digraph $\mathcal{D}(L)$ is the union of cycles, observability and reconstructibility are equivalent properties. In the general case, it turns out that reconstructibility is equivalent to the fact that all states that belong to the cycles are distinguishable one from the other.

Theorem 2: Given a $\mathrm{BN}$ (3), the following facts are equivalent:

i) the $\mathrm{BN}$ is reconstructible;

ii) the reduced $\mathrm{BN}$, obtained from (3) by considering only the states that belong to some cycle ${ }^{3}$, is observable;

iii) for every pair of distinct periodic state trajectories of the same minimal period $k$, described by the two ordered $k$-tuples

$$
\left(\mathbf{x}_{1}, \mathbf{x}_{2}, \ldots, \mathbf{x}_{k}\right) \neq\left(\overline{\mathbf{x}}_{1}, \overline{\mathbf{x}}_{2}, \ldots, \overline{\mathbf{x}}_{k}\right),
$$

the corresponding output trajectories are periodic of (minimal) period $k$ and described by two different $k$-tuples, i.e.

$$
\left(H \mathbf{x}_{1}, H \mathbf{x}_{2}, \ldots, H \mathbf{x}_{k}\right) \neq\left(H \overline{\mathbf{x}}_{1}, H \overline{\mathbf{x}}_{2}, \ldots, H \overline{\mathbf{x}}_{k}\right) .
$$

Moreover, when the $\mathrm{BN}$ is reconstructible, $T_{\min } \leq T_{r}+\bar{N}-1$, where $T_{r}$ is the minimum number of steps after which the state of the BN surely belongs to a cycle, while $\bar{N}$ is the number of states of the $\mathrm{BN}$ that belong to a cycle, i.e.

$$
\begin{aligned}
T_{r} & :=\min \left\{t \in \mathbb{Z}_{+}: L^{t} \mathbf{x} \in \cup_{i=1}^{r} \mathcal{Z}_{i}, \forall \mathbf{x} \in \mathcal{L}_{N}\right\} \mid, \\
\bar{N} & :=\left|\left\{\mathbf{x} \in \mathcal{L}_{N}: \mathbf{x} \in \mathcal{Z}_{i}, \exists i \in[1, r]\right\}\right|,
\end{aligned}
$$

$\mathcal{Z}_{i}, i \in[1, r]$, being the distinct cycles of the $\mathrm{BN}$.

Proof: i) $\Rightarrow$ iii) If iii) were not satisfied, there would be two distinct initial states, $\mathbf{x}_{1}$ and $\overline{\mathbf{x}}_{1}$, that produce two distinct periodic state trajectories of the same minimal period and the same output trajectory. Hence at every time $t$, we would not be able to distinguish $\mathbf{x}(t)$ from $\overline{\mathbf{x}}(t)$, the states reached at time $t$ starting from $\mathbf{x}_{1}$ and from $\overline{\mathbf{x}}_{1}$, respectively. So, for every choice of $T$, from the output in $[0, T]$, we could not determine the state at time $T$.

iii) $\Rightarrow$ ii) Follows from Theorem 1 .

ii) $\Rightarrow$ i) Set $T:=T_{r}+\bar{N}-1$. Every state trajectory $\mathbf{x}(t)$ is surely periodic for $t \geq T_{r}$, which amounts to saying that $\mathbf{x}\left(T_{r}\right)$ belongs to some cycle $\mathcal{Z}_{i}, i \in[1, r]$. If $\bar{N}=1$, namely the reduced $\mathrm{BN}$ is one-dimensional, then clearly reconstructibility holds for $T=T_{r}$. So, assume now that $\bar{N}>1$ (and hence $\rho$, the number of nonzero rows of $H$, is not smaller than 2). By the observability of the reduced $\mathrm{BN}$ and Lemma 1, we can claim that upon observing $\mathbf{y}(t), t \in\left[T_{r}, T_{r}+\bar{N}-2\right]$, we can identify the state $\mathbf{x}\left(T_{r}\right)$. So, in particular, we can determine $\mathbf{x}\left(T_{r}+\bar{N}-1\right)$. This ensures that the $\mathrm{BN}$ is reconstructible and that $T_{\min } \leq T_{r}+\bar{N}-1$.

\footnotetext{
${ }^{3}$ Note that the definition is well posed, since cycles are invariant sets, and indeed each state belonging to a cycle can only access one and only one state
} belonging to the same cycle. 
Remark 3: By referring to the notation adopted in Proposition 3 , it is easy to see that $T_{r}$ is the largest of the nilpoten indices of the matrices $W_{\nu}, \nu \in[1, r]$, and hence

$$
T_{r}+\bar{N}-1 \leq\left(\max _{i \in[1, r]}\left(n_{i}-k_{i}\right)\right)+\sum_{i=1}^{r} k_{i}-1
$$

Also, if we assume again that $H$ is block-partitioned as Remark 2, the BN (3) is reconstructible if and only if

i) each block $H_{\nu c}$ cannot be seen as the juxtaposition two or more copies of the same block, i.e. $H_{\nu c}$ $\left[\begin{array}{llll}\Phi_{\nu} & \Phi_{\nu} & \ldots & \Phi_{\nu}\end{array}\right]$

ii) if $\ell \neq d$, the blocks $H_{\ell c}$ and $H_{d c}$ are distinct a cannot be obtained one from the other by means of cyc permutations of the columns, i.e. $\nexists$ a cyclic matrix $C$ a $h \in \mathbb{Z}_{+}$such that $H_{d c}=H_{\ell c} C^{h}$.

Remark 4: To conclude the section, we want to comme on the feasibility of the observability and reconstructibil: tests provided by Theorems 1 and 2 . To test reconstructibility, we can proceed as follows: we first determine $T_{r}$ as ${ }^{4}$

$$
T_{r}=\min \left\{t \in \mathbb{Z}_{+}: \operatorname{rank} L^{t}=\operatorname{rank} L^{t+1}\right\}
$$

and let $I$ be the set of indices of the nonzero rows in $L^{T_{r}}$. Such indices correspond to the states that belong to some cycle or are equilibrium points. If $|I|=1$, the $\mathrm{BN}$ is obviously reconstructible. If $|I|>1$, the $\mathrm{BN}$ is reconstructible if and only if the output trajectories corresponding to the initial states $\delta_{N}^{i}, i \in I$, are distinct. This amounts to checking that all the columns of

$$
\mathcal{O}_{\bar{N}-1}=\left[\begin{array}{c}
H \\
H L \\
\vdots \\
H L^{\bar{N}-2}
\end{array}\right], \quad \text { with } \bar{N}:=|I|,
$$

corresponding to the indices in $I$ are distinct.

On the other hand, by Theorem 1 , a $\mathrm{BN}$ is observable if and only if it is reconstructible and the matrix $\left[\begin{array}{c}L \\ H\end{array}\right]$ has all distinct columns.

The following example shows that the bound $T_{\min } \leq T_{r}+$ $\bar{N}-1$ is tight.

Example 2: Consider the $\mathrm{BN}$ (3) with

$$
\begin{aligned}
& L=\left[\begin{array}{llllllll}
\delta_{8}^{2} & \delta_{8}^{3} & \delta_{8}^{4} & \delta_{8}^{4} & \delta_{8}^{6} & \delta_{8}^{7} & \delta_{8}^{8} & \delta_{8}^{4}
\end{array}\right] \\
& H=\left[\begin{array}{llllllll}
\delta_{2}^{1} & \delta_{2}^{1} & \delta_{2}^{1} & \delta_{2}^{1} & \delta_{2}^{1} & \delta_{2}^{1} & \delta_{2}^{1} & \delta_{2}^{1}
\end{array}\right]
\end{aligned}
$$

\footnotetext{
${ }^{4}$ For Boolean matrices three notions of rank have been defined: column rank, row rank and Schein rank [15], however for the semigroup of Boolean matrices $\mathcal{L}_{N \times N}$ these three concepts coincide and they can be simply regarded as the maximum number of distinct rows (or columns) in the matrix.
}

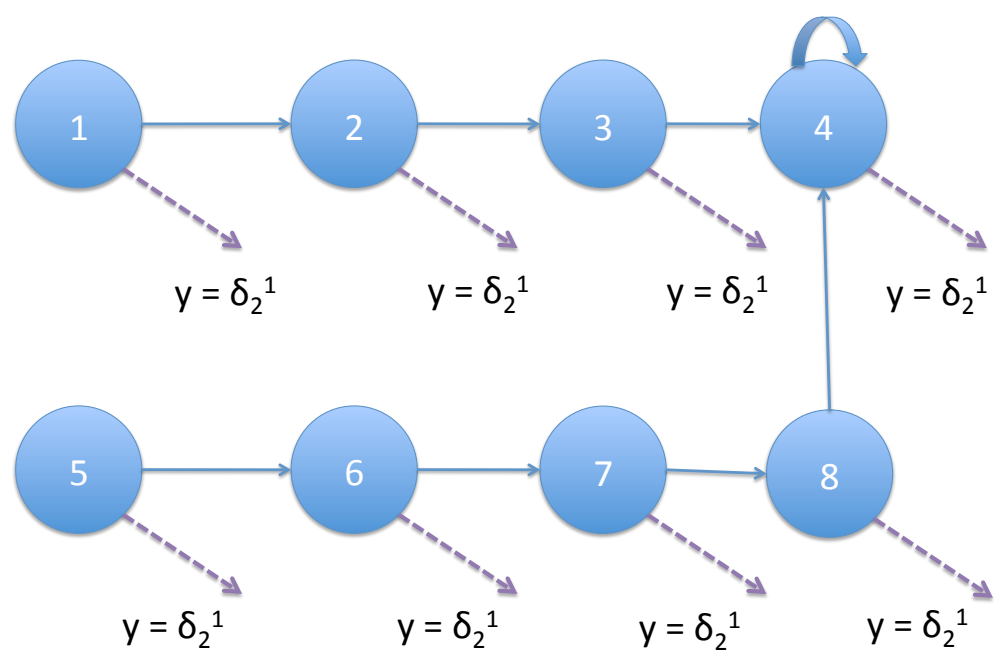

FIG. 2. Digraph corresponding to the BN of Example 2.

It is easy to see that the $\mathrm{BN}$ is reconstructible, since there is only one vertex (vertex 4$)$ belonging to a cycle $(\bar{N}=1)$. Also, we easily see that $T_{r}=4$ (the distance from 5 to 4 ). We want to prove that the smallest time instant $T$ at which we are able to identify the state $\mathbf{x}(T)$ from the output evolution $\mathbf{y}(t), t \in[0, T]$, is just $T_{r}+\bar{N}-1=4$. This follows trivially from the fact that there is no chance to identify the current state from the output evolution up to that time, unless it is clear that we have reached the state $\delta_{8}^{4}$.

Remark 5: Reconstructibility may be related to the indistinguishability classes $\mathcal{C}_{\sim, k}, k \in \mathbb{N}$, that we introduced in the previous section. Indeed, it is easy to see that a $\mathrm{BN}$ is reconstructible if and only if there exists $\bar{T} \in \mathbb{Z}_{+}$such that in every indistinguishability class $\mathcal{K}_{i}$ of $\mathcal{C}_{\sim, \bar{T}}$ there is only one state that belongs to a cycle. This is also equivalent to saying that there exists $T \in \mathbb{Z}_{+}$such that, for every class $\mathcal{K}_{i}$ of $\mathcal{C}_{\sim, T}$, the set $L^{T} \mathcal{K}_{i}$ consists of a single state. Clearly, $\bar{T}$ is related to the observability index of the reduced $\mathrm{BN}$, while $T$ to the reconstructibility index of the $\mathrm{BN}$.

\section{OBSERVABility of BoOlean CONTROL Networks}

A Boolean Control Network (BCN) is described by the following equations

$$
\begin{aligned}
X(t+1) & =f(X(t), U(t)) \\
Y(t) & =h(X(t)), \quad t \in \mathbb{Z}_{+},
\end{aligned}
$$

where $X(t), U(t)$ and $Y(t)$ denote the $n$-dimensional state variable, the $m$-dimensional input and the $p$-dimensional output at time $t$, taking values in $\mathcal{B}^{n}, \mathcal{B}^{m}$ and $\mathcal{B}^{p}$, respectively. $f$ and $h$ are logic functions, i.e. $f: \mathcal{B}^{n} \times \mathcal{B}^{m} \rightarrow \mathcal{B}^{n}$ and $h: \mathcal{B}^{n} \rightarrow \mathcal{B}^{p}$. By resorting to the semi-tensor product $\ltimes$, the BCN (10) can be described [8] as

$$
\begin{aligned}
\mathbf{x}(t+1) & =L \ltimes \mathbf{u}(t) \ltimes \mathbf{x}(t), \\
\mathbf{y}(t) & =H \ltimes \mathbf{x}(t)=H \mathbf{x}(t), \quad t \in \mathbb{Z}_{+},
\end{aligned}
$$


where $\mathbf{x}(t) \in \mathcal{L}_{N}, \mathbf{u}(t) \in \mathcal{L}_{M}$ and $\mathbf{y}(t) \in \mathcal{L}_{P}$, with $N:=$ $2^{n}, M:=2^{m}$ and $P:=2^{p} . L \in \mathcal{L}_{N \times N M}$ and $H \in \mathcal{L}_{P \times N}$ are matrices whose columns are canonical vectors of size $N$ and $P$, respectively. For every choice of the input variable at $t$, namely for every $\mathbf{u}(t)=\delta_{M}^{j}, L \ltimes \mathbf{u}(t)=: L_{j}$ is a matrix in $\mathcal{L}_{N \times N}$. So, we can think of the state equation of the BCN (11) as a Boolean switched system,

$$
\mathbf{x}(t+1)=L_{\sigma(t)} \mathbf{x}(t), \quad t \in \mathbb{Z}_{+},
$$

where $\sigma(t), t \in \mathbb{Z}_{+}$, is a switching sequence taking values in $[1, M]$. For every $j \in[1, M]$, the BN

$$
\mathbf{x}(t+1)=L_{j} \mathbf{x}(t), \quad t \in \mathbb{Z}_{+},
$$

represents the $j$ th subsystem of (12).

Definition 3: A BCN (3) is observable if for any two initial states $\mathbf{x}_{1}=\delta_{2^{n}}^{i}$ and $\mathbf{x}_{2}=\delta_{2^{n}}^{j}, i \neq j$, and any input sequence $\mathbf{u}(t), t \in \mathbb{Z}_{+}$, the output evolutions $\mathbf{y}_{1}(t)$ and $\mathbf{y}_{2}(t), t \in \mathbb{Z}_{+}$, are distinct.

In [8], Chapter 16, a different notion of observability has been introduced, stating that a BCN is observable if for any two states $\mathbf{x}_{1}=\delta_{2^{n}}^{i}$ and $\mathbf{x}_{2}=\delta_{2^{n}}^{j}, i \neq j$, there exists an input sequence $\mathbf{u}_{i j}(t), t \in \mathbb{Z}_{+}$, such that the corresponding output evolutions $\mathbf{y}_{1}(t)$ and $\mathbf{y}_{2}(t), t \in \mathbb{Z}_{+}$, are distinct. In [13] (page 177), these two notions are referred to as "initialstate determinability in the strong sense" and "initial-state determinability in the wide sense", respectively. For linear state-space models these two notions are equivalent, since the observability problem can always be reduced to that of determining the initial condition that generated the free state evolution. This is not the case when dealing BCNs. Indeed, observability given in Definition 3 implies the notion of observability given in [8], while the converse is not true, due to the fact that it is not possible to express the (state and) output dynamics as the sum of a free and a forced evolution, and hence remove the forced component.

Observability property considered in this paper allows to determine the initial condition of a $\mathrm{BCN}$ when it evolves under the effect of an arbitrary input. This is consistent with the natural requirement of estimating the initial (or the current) state of a $\mathrm{BCN}$, driven by an arbitrary control sequence, and hence under regular working conditions, and not by means of an ad hoc experiment. On the other hand, the observability property introduced in [8] allows to identify the initial condition of the BN only by carrying on multiple parallel experiments, as clarified in [13], and not by making use of the observation of a single pair of corresponding input/output evolutions.

To address observability, we introduce some new notation. Since for BCNs the state evolution depends on both the state and the input we will consider instead of just state trajectories, state-input trajectories, described by the pairs $(\mathbf{x}(t), \mathbf{u}(t)), t \in$ $\mathbb{Z}_{+}$. Also, we say that a state-input trajectory is periodic of (minimal) period $k \in \mathbb{N}$ if $(\mathbf{x}(t), \mathbf{u}(t))=(\mathbf{x}(t+k), \mathbf{u}(t+$ $k)$ ), $\forall t \in \mathbb{Z}_{+}$, (and there is no smaller $k$ for which this is true). A periodic state-input trajectory of period $k \in \mathbb{N}$ is described by an ordered $k$-tuple of pairs

$$
\left(\left(\mathbf{x}_{1}, \mathbf{u}_{1}\right),\left(\mathbf{x}_{2}, \mathbf{u}_{2}\right), \ldots,\left(\mathbf{x}_{k}, \mathbf{u}_{k}\right)\right),
$$

where by this notation we mean that

$\mathbf{x}_{\ell+1}=L \ltimes \mathbf{u}_{\ell} \ltimes \mathbf{x}_{\ell}, \forall \ell \in[1, k-1]$, and $\mathbf{x}_{1}=L \ltimes \mathbf{u}_{k} \ltimes \mathbf{x}_{k}$,

and, as before, we say that such a $k$-tuple is irreducible if it cannot be expressed as the repetition of shorter tuples. This amounts to saying that $k$ is the minimal period.

\section{Theorem 3: A BCN (11) is observable if and only if}

i) [distinguishability of states before state merging] for every $\mathbf{x}_{1}, \mathbf{x}_{2} \in \mathcal{L}_{N}$ and for every $\mathbf{u} \in \mathcal{L}_{M}$, conditions $\left(\mathbf{x}_{1}, \mathbf{u}\right) \neq\left(\mathbf{x}_{2}, \mathbf{u}\right)$ and $L \ltimes \mathbf{u} \ltimes \mathbf{x}_{1}=L \ltimes \mathbf{u} \ltimes \mathbf{x}_{2}$ imply $H \mathbf{x}_{1} \neq H \mathbf{x}_{2}$;

ii) [distinguishability of states belonging to (the same or different) cycles] for every pair of distinct periodic stateinput trajectories of the same minimal period $k$ and described by the two distinct ordered $k$-tuples

$$
\left(\left(\mathbf{x}_{1}, \mathbf{u}_{1}\right),\left(\mathbf{x}_{2}, \mathbf{u}_{2}\right), \ldots,\left(\mathbf{x}_{k}, \mathbf{u}_{k}\right)\right) \neq\left(\left(\overline{\mathbf{x}}_{1}, \mathbf{u}_{1}\right),\left(\overline{\mathbf{x}}_{2}, \mathbf{u}_{2}\right), \ldots,\left(\overline{\mathbf{x}}_{k}, \mathbf{u}\right.\right.
$$

the corresponding output trajectories are periodic of (minimal) period $k$ and described by two different $k$-tuples, i.e.

$$
\left(H \mathbf{x}_{1}, H \mathbf{x}_{2}, \ldots, H \mathbf{x}_{k}\right) \neq\left(H \overline{\mathbf{x}}_{1}, H \overline{\mathbf{x}}_{2}, \ldots, H \overline{\mathbf{x}}_{k}\right) .
$$

Proof: [Necessity] If condition i) was not satisfied for two initial states $\mathbf{x}_{1}(0)=\mathbf{x}_{1} \neq \mathbf{x}_{2}(0)=\mathbf{x}_{2}$ and some $\mathbf{u} \in$ $\mathcal{L}_{M}$, any input sequence $\mathbf{u}(t), t \in \mathbb{Z}_{+}$, with $\mathbf{u}(0)=\mathbf{u}$, would produce two state trajectories satisfying $\mathbf{x}_{1}(t)=\mathbf{x}_{2}(t)$ for $t \geq 1$, and the corresponding output trajectories, $\mathbf{y}_{1}(t)$ and $\mathbf{y}_{2}(t)$, would coincide for every $t \in \mathbb{Z}_{+}$. Hence the two states would be indistinguishable, a contradiction.

On the other hand, if condition ii) was not satisfied, there would be two distinct initial states, $\mathbf{x}_{1}$ and $\overline{\mathbf{x}}_{1}$, that generate the same periodic output trajectory, corresponding to the periodic input

$$
\mathbf{u}(t)= \begin{cases}\mathbf{u}_{t+1}, & \text { for } t \in[0, k-1] \\ \mathbf{u}(t-k), & \text { for } t \geq k\end{cases}
$$

where the vectors $\mathbf{u}_{1}, \mathbf{u}_{2}, \ldots, \mathbf{u}_{k}$ have been introduced in equation (14). So, again, observability would be contradicted.

[Sufficiency] We want to prove that if conditions i) and ii) hold, then the BCN is observable. Suppose it is not. Then two distinct states $\mathbf{x}(0)$ and $\overline{\mathbf{x}}(0)$ and some input sequence $\mathbf{u}(t), t \in \mathbb{Z}_{+}$, could be found such that the corresponding output trajectories, $\mathbf{y}(t)$ and $\overline{\mathbf{y}}(t)$, coincide for every $t \in \mathbb{Z}_{+}$. Let $\mathbf{x}(t)$ and $\overline{\mathbf{x}}(t), t \in \mathbb{Z}_{+}$, be the state trajectories corresponding to the given initial states and input. If there would be $t^{\prime} \in \mathbb{Z}_{+}$such that the two state trajectories coincide at $t^{\prime}+1$, i.e. $\mathbf{x}\left(t^{\prime}+1\right)=\overline{\mathbf{x}}\left(t^{\prime}+1\right)$, this would contradict assumption i) for the two state-input pairs $\left(\mathbf{x}\left(t^{\prime}\right), \mathbf{u}\left(t^{\prime}\right)\right)$ and $\left(\overline{\mathbf{x}}\left(t^{\prime}\right), \mathbf{u}\left(t^{\prime}\right)\right)$. So, we now assume that, at every time $t \in \mathbb{Z}_{+}, \mathbf{x}(t) \neq \overline{\mathbf{x}}(t)$. Consider the sequence of triples $(\mathbf{x}(t), \overline{\mathbf{x}}(t), \mathbf{u}(t)), t \in \mathbb{Z}_{+}$. Since all such triples take values in the finite set $\mathcal{L}_{N} \times$ $\mathcal{L}_{N} \times \mathcal{L}_{M}$, there exist $t_{m}, k \in \mathbb{Z}_{+}, k>0$, such that $\left(\mathbf{x}\left(t_{m}\right), \overline{\mathbf{x}}\left(t_{m}\right), \mathbf{u}\left(t_{m}\right)\right)=\left(\mathbf{x}\left(t_{m}+k\right), \overline{\mathbf{x}}\left(t_{m}+k\right), \mathbf{u}\left(t_{m}+k\right)\right)$, 
and we may always select the smallest such $k$. Clearly if we replace the original sequence $\mathbf{u}(t)$ with the sequence

$$
\tilde{\mathbf{u}}(t):= \begin{cases}\mathbf{u}(t), & 0 \leq t \leq t_{m}+k-1 \\ \tilde{\mathbf{u}}(t-k), & t \geq t_{m}+k\end{cases}
$$

we still get two output trajectories completely identical (a identical to the original ones till $\left.t=t_{m}+k-1\right)$. But tl means that the two distinct irreducible $k$-tuples

$$
\begin{array}{r}
\left(\left(\mathbf{x}\left(t_{m}\right), \mathbf{u}\left(t_{m}\right)\right), \ldots,\left(\mathbf{x}\left(t_{m}+k-1\right), \mathbf{u}\left(t_{m}+k-1\right)\right)\right) \\
\left(\left(\overline{\mathbf{x}}\left(t_{m}\right), \mathbf{u}\left(t_{m}\right)\right), \ldots,\left(\overline{\mathbf{x}}\left(t_{m}+k-1\right), \mathbf{u}\left(t_{m}+k-1\right)\right)\right)
\end{array}
$$

generate the same output $k$-tuple, as

$$
\left(\mathbf{y}\left(t_{m}\right), \ldots, \mathbf{y}\left(t_{m}+k-1\right)\right)=\left(\overline{\mathbf{y}}\left(t_{m}\right), \ldots, \overline{\mathbf{y}}\left(t_{m}+k-1\right)\right)
$$

This contradicts condition ii).

We illustrate the previous conditions by means of an example.

Example 3: Consider a BCN (11) and suppose that ${ }^{5}$

$$
\begin{aligned}
& L_{1}:=L \ltimes \delta_{2}^{1}=\left[\begin{array}{lllll}
1 & 0 & 0 & 0 & 0 \\
0 & 0 & 0 & 0 & 0 \\
0 & 0 & 1 & 0 & 0 \\
0 & 1 & 0 & 0 & 1 \\
0 & 0 & 0 & 1 & 0
\end{array}\right]=\left[\begin{array}{llll}
\delta_{5}^{1} & \delta_{5}^{4} & \delta_{5}^{3} & \delta_{5}^{5}
\end{array}\right. \\
& L_{2}:=L \ltimes \delta_{2}^{2}=\left[\begin{array}{lllll}
0 & 0 & 0 & 0 & 0 \\
1 & 0 & 0 & 0 & 0 \\
0 & 1 & 1 & 0 & 0 \\
0 & 0 & 0 & 1 & 1 \\
0 & 0 & 0 & 0 & 0
\end{array}\right]=\left[\begin{array}{llll}
\delta_{5}^{2} & \delta_{5}^{3} & \delta_{5}^{3} & \delta_{5}^{4}
\end{array}\right. \\
& H=\left[\begin{array}{lllll}
1 & 1 & 0 & 1 & 0 \\
0 & 0 & 1 & 0 & 1
\end{array}\right]=\left[\begin{array}{lllll}
\delta_{2}^{1} & \delta_{2}^{1} & \delta_{2}^{2} & \delta_{2}^{1} & \delta_{2}^{2}
\end{array}\right]
\end{aligned}
$$

The $\mathrm{BCN}$ can be represented by the following digraph, obtained by overlapping the two digraphs $\mathcal{D}\left(L_{1}\right)$ and $\mathcal{D}\left(L_{2}\right)$. (Blue) arcs labelled by $\mathbf{u}=\delta^{1}$ belong to $\mathcal{D}\left(L_{1}\right)$, while (red) arcs labelled by $\mathbf{u}=\delta^{2}$ belong to $\mathcal{D}\left(L_{2}\right)$. As for $\mathrm{BNs}$, the outgoing (dashed) arrows indicate for each state the corresponding output value $\left(\mathbf{y}=\delta^{1}\right.$ or $\left.\mathbf{y}=\delta^{2}\right)$.

\footnotetext{
${ }^{5}$ Note that the size of the system matrices $L_{i}$ is $N=5$ which is not a power of 2 , but the analysis is not affected by this fact.
}

FIG. 3. Digraph corresponding to the BCN of Example 3.

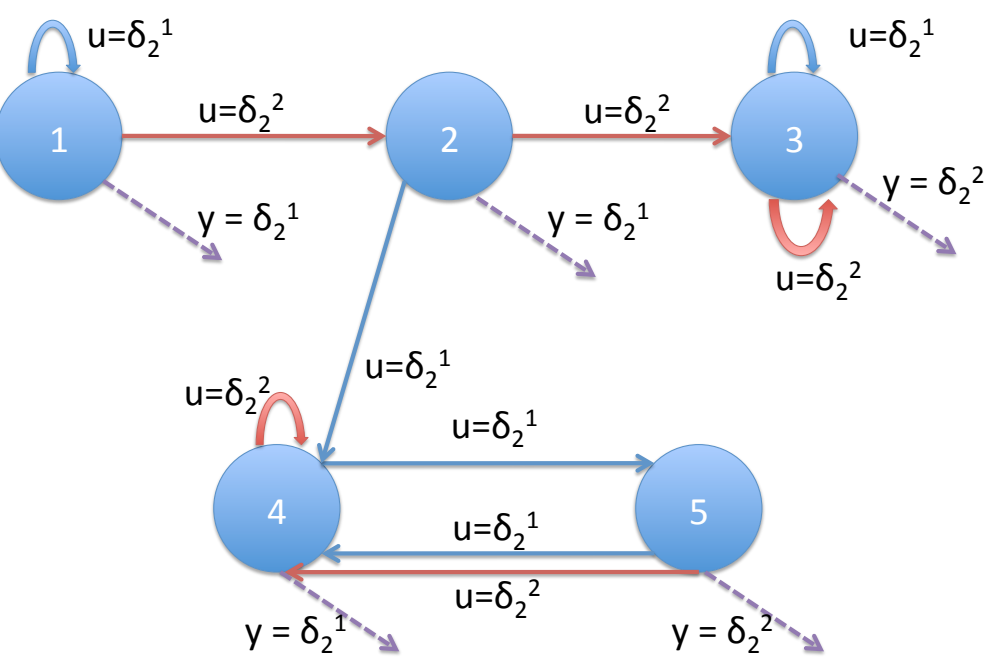

It is a matter of simple computation to verify that both conditions of Theorem 3 are verified. Specifically:

i) $L_{1} \delta_{5}^{2}=L_{1} \delta_{5}^{5}=\delta_{5}^{4}$ but $H \delta_{5}^{2} \neq H \delta_{5}^{5}$;

$L_{2} \delta_{5}^{2}=L_{2} \delta_{5}^{3}=\delta_{5}^{3}$ but $H \delta_{5}^{2} \neq H \delta_{5}^{3}$;

$L_{2} \delta_{5}^{4}=L_{2} \delta_{5}^{5}=\delta_{5}^{4}$ but $H \delta_{5}^{4} \neq H \delta_{5}^{5}$;

ii) there are two cycles of length 1 corresponding to the input $\delta_{2}^{1}$, namely self loops, but they correspond to two states that generate different outputs; similarly, there are two cycles of length 1 corresponding to the input $\delta_{2}^{2}$, but they generate different output trajectories. Finally, there are two distinct ordered 2-tuples that correspond to 2 periodic input-state trajectories, namely

$$
\left(\left(\delta_{5}^{4}, \delta_{2}^{1}\right),\left(\delta_{5}^{5}, \delta_{2}^{1}\right)\right) \neq\left(\left(\delta_{5}^{5}, \delta_{2}^{1}\right),\left(\delta_{5}^{4}, \delta_{2}^{1}\right)\right),
$$

\section{$\left.\delta_{5}^{4}\right]$}

and they generate two different periodic output trajectories

$$
\left(\delta_{2}^{1}, \delta_{2}^{2}\right) \neq\left(\delta_{2}^{2}, \delta_{2}^{1}\right) .
$$

Consequently, the $\mathrm{BCN}$ is observable.

$\left.\delta_{5}^{4}\right]$,

Remark 6: Observability corresponds to the possibility of uniquely determining the initial state of the $\mathrm{BCN}$, once the input sequence $\mathbf{u}(t), t \in \mathbb{Z}_{+}$, and the corresponding output $\mathbf{y}(t), t \in \mathbb{Z}_{+}$, are known. As we have seen in section II, the initial state of an observable $\mathrm{BN}$ can be retrieved from the output samples $\mathbf{y}(t), t \in[0, N-2]$. Something similar happens with observable BCNs, as indeed, the knowledge of $\mathbf{u}(t)$ and $\mathbf{y}(t)$ for $t \in\left[0, N^{2}\right]$ is sufficient to uniquely determine $\mathbf{x}(0)$. To show this, it is sufficient to prove that if there exist an input sequence $\mathbf{u}(t), t \in \mathbb{Z}_{+}$, and two states, say $\mathbf{x}_{1}$ and $\mathbf{x}_{2}$, such that $\mathbf{y}_{1}(t)=\mathbf{y}_{2}(t)$ for every $t \in\left[0, N^{2}\right]$, then there exists an input $\tilde{\mathbf{u}}(t), t \in \mathbb{Z}_{+}$, that makes the states $\mathbf{x}_{1}$ and $\mathbf{x}_{2}$ indistinguishable (i.e. $\tilde{\mathbf{y}}_{1}(t)=\tilde{\mathbf{y}}_{2}(t)$ for every $\left.t \in \mathbb{Z}_{+}\right)$. Indeed, consider the sequence of pairs $\left(\mathbf{x}_{1}(t), \mathbf{x}_{2}(t)\right)$ generated from $\left(\mathbf{x}_{1}(0), \mathbf{x}_{2}(0)\right)=\left(\mathbf{x}_{1}, \mathbf{x}_{2}\right)$ corresponding to $\mathbf{u}(t)$. Clearly, since at each time $t$ the pair $\left(\mathbf{x}_{1}(t), \mathbf{x}_{2}(t)\right)$ 
belongs to the finite set $\mathcal{L}_{N} \times \mathcal{L}_{N}$, a cardinality argument ensures that there exist $t_{m}$ and $k$, with $0 \leq t_{m}<t_{m}+k \leq N^{2}$, such that $\left(\mathbf{x}_{1}\left(t_{m}\right), \mathbf{x}_{2}\left(t_{m}\right)\right)=\left(\mathbf{x}_{1}\left(t_{m}+k\right), \mathbf{x}_{2}\left(t_{m}+k\right)\right)$. So, as in the proof of Theorem 3 , the input sequence

$$
\tilde{\mathbf{u}}(t):= \begin{cases}\mathbf{u}(t), & 0 \leq t \leq t_{m}+k-1 \\ \tilde{\mathbf{u}}(t-k), & t \geq t_{m}+k\end{cases}
$$

would produce two identical output sequences.

As a matter of fact, the upper bound of $N^{2}$ can be significantly improved. To this end, we may ask how large the parameter $T$ can be chosen, so that two distinct initial states $\mathbf{x}_{1}$ and $\mathbf{x}_{2}$ generate two output sequences $\mathbf{y}_{1}(t)$ and $\mathbf{y}_{2}(t)$ that coincide for every $t \in[0, T]$, without contradicting observability. We have already noticed that all pairs $\left(\mathbf{x}_{1}(t), \mathbf{x}_{2}(t)\right), t \in$ $[0, T]$, must be distinct. On the other hand, if at some time instant $t^{\prime}, \mathbf{x}_{1}\left(t^{\prime}\right)=\mathbf{x}_{2}\left(t^{\prime}\right)$, then the output trajectories $\mathbf{y}_{1}(t)$ and $\mathbf{y}_{2}(t)$ would necessarily coincide from $t^{\prime}$ onward. Finally, if there exist $t_{m}$ and $k$, with $0 \leq t_{m}<t_{m}+k \leq T$, such that $\mathbf{x}_{1}\left(t_{m}\right)=\mathbf{x}_{2}\left(t_{m}+k\right)$ and $\mathbf{x}_{2}\left(t_{m}\right)=\mathbf{x}_{1}\left(t_{m}+k\right)$, then it is clear that the input sequence (15) would again produce two identical output sequences. So, in the time interval $[0, T]$ all pairs $\left(\mathbf{x}_{1}(t), \mathbf{x}_{2}(t)\right), t \in \mathbb{Z}_{+}$, must be distinct, cannot have two entries of same value and cannot appear in one order and in the reversed one. This implies that

$$
T+1 \leq N^{2}-N-\sum_{i=1}^{N-1} i=\sum_{i=1}^{N-1} i=\frac{N(N-1)}{2}=: N^{*}
$$

and hence

$$
T \leq \frac{(N+1)(N-2)}{2}
$$

Remark 7: To conclude we want to compare the characterization of the observability property given in [8] (see Theorem 9.4, pages 228-229) with a similar one that can be obtained for the observability notion used in this paper. To this end we denote by

$$
\mathcal{O}_{u, h}:=\left[\begin{array}{c}
H \\
H L_{i_{0}} \\
H L_{i_{1}} L_{i_{0}} \\
\vdots \\
H L_{i_{h-2}} \ldots L_{i_{1}}
\end{array}\right]
$$

the observability matrix in $h$ steps corresponding to the input sequence

$$
\mathbf{u}(0)=\delta_{M}^{i_{0}}, \quad \mathbf{u}(1)=\delta_{M}^{i_{1}}, \quad \ldots \quad, \mathbf{u}(h-2)=\delta_{M}^{i_{h-2}} .
$$

Clearly, the $i$ th column of $\mathcal{O}_{u, h}$ provides the output sequence $\mathbf{y}(t), t \in[0, h-1]$, corresponding to the aforementioned input and the initial condition $\mathbf{x}(0)=\delta_{N}^{i}$. By the previous remark, observability here addressed is equivalent to the fact that all the matrices $\mathcal{O}_{u, N^{*}}$, for every choice of the input sequence, have distinct columns. The characterization given in Theorem 9.4 of [8] can be equivalently restated by saying that the $\mathrm{BCN}$ is observable in the sense of [8] if and only if the matrix obtained by piling up all the matrices $\mathcal{O}_{u, N^{*}}$ has distinct columns.

\section{RECONSTRUCTIBILITY OF BOOLEAN CONTROL NETWORKS}

Definition 4: A BCN (11) is said to be reconstructible if there exists $T \in \mathbb{Z}_{+}$such that, for every input sequence, the simultaneous knowledge ${ }^{6}$ of the input and the output trajectories, $\mathbf{u}(t)$ and $\mathbf{y}(t), t \in[0, T]$, allows to uniquely determine $\mathbf{x}(T)$.

The characterization of reconstructibility is similar to the one obtained for BNs, even if its proof is more challenging.

Theorem 4: A BCN (11) is reconstructible if and only if for every pair of distinct periodic state-input trajectories of the same minimal period $k$ and described by the two ordered $k$-tuples

$$
\left(\left(\mathbf{x}_{1}, \mathbf{u}_{1}\right), \ldots,\left(\mathbf{x}_{k}, \mathbf{u}_{k}\right)\right) \neq\left(\left(\overline{\mathbf{x}}_{1}, \mathbf{u}_{1}\right), \ldots,\left(\overline{\mathbf{x}}_{k}, \mathbf{u}_{k}\right)\right),
$$

the corresponding output trajectories are periodic of (minimal) period $k$ and described by two different $k$-tuples, i.e.

$$
\left(H \mathbf{x}_{1}, H \mathbf{x}_{2}, \ldots, H \mathbf{x}_{k}\right) \neq\left(H \overline{\mathbf{x}}_{1}, H \overline{\mathbf{x}}_{2}, \ldots, H \overline{\mathbf{x}}_{k}\right) .
$$

Proof: [Necessity] If the condition would not be satisfied, the two distinct periodic state trajectories generated from $\mathbf{x}_{1}$ and $\overline{\mathbf{x}}_{1}$, corresponding to the periodic input sequence

$$
\mathbf{u}(t)= \begin{cases}\mathbf{u}_{t+1}, & \text { for } t \in[0, k-1], \\ \mathbf{u}(t-k), & \text { for } t \geq k,\end{cases}
$$

would produce to the same periodic output trajectory. So, all pairs of states along the two state trajectories would be distinct and indistinguishable, and reconstructibility would be contradicted.

[Sufficiency] Suppose, by contradiction, that the BCN is not reconstructible. This means that there exists a pair of corresponding input and output trajectories $\mathbf{u}(t)$ and $\mathbf{y}(t), t \in \mathbb{Z}_{+}$, such that for every $T \in \mathbb{Z}_{+}$the set $\mathcal{X}_{T}$ of all states that are compatible at time $T$ with the given input and output has cardinality greater than 1 . It is clear that

$$
\left|\mathcal{X}_{0}\right| \geq\left|\mathcal{X}_{1}\right| \geq \ldots \geq\left|\mathcal{X}_{t}\right| \geq \ldots,
$$

and there exists $\bar{T}$ such that $C:=\left|\mathcal{X}_{\bar{T}}\right|=\left|\mathcal{X}_{\bar{T}+t}\right|$ for every $t \geq 0$, and $C>1$. Again, by a cardinality argument, we can claim that there exist two states $\mathbf{x}_{1}, \overline{\mathbf{x}}_{1}$ and two integers $t_{m} \geq \bar{T}$ and $k>0$ such that

$$
\mathbf{x}_{1}, \overline{\mathbf{x}}_{1} \in \mathcal{X}_{t_{m}} \cap \mathcal{X}_{t_{m}+k}
$$

and the two distinct periodic sequences corresponding to the two irreducible $k$-tuples

$$
\begin{aligned}
& \left(\left(\mathbf{x}_{1}\left(t_{m}\right), \mathbf{u}\left(t_{m}\right)\right), \ldots,\left(\mathbf{x}_{1}\left(t_{m}+k-1\right), \mathbf{u}\left(t_{m}+k-1\right)\right),\right. \\
& \left(\left(\overline{\mathbf{x}}_{1}\left(t_{m}\right), \mathbf{u}\left(t_{m}\right)\right), \ldots,\left(\overline{\mathbf{x}}_{1}\left(t_{m}+k-1\right), \mathbf{u}\left(t_{m}+k-1\right)\right),\right.
\end{aligned}
$$

with $\mathbf{x}_{1}\left(t_{m}\right)=\mathbf{x}_{1}$ and $\overline{\mathbf{x}}_{1}\left(t_{m}\right)=\overline{\mathbf{x}}_{1}$, generate the same periodic output, since

$$
\left(H \mathbf{x}_{1}\left(t_{m}\right), H \mathbf{x}_{1}\left(t_{m}+1\right), \ldots, H \mathbf{x}_{1}\left(t_{m}+k-1\right)=\right.
$$

${ }^{6}$ Due to the structure of the equations (11), in order to reconstruct the final state at $t=T$ the input value at $t=T$ is unnecessary. We chose this definition just for the sake of simplicity. 


$$
\begin{gathered}
\left(\mathbf{y}\left(t_{m}\right), \mathbf{y}\left(t_{m}+1\right), \ldots, \mathbf{y}\left(t_{m}+k-1\right)\right)= \\
\left(H \overline{\mathbf{x}}_{1}\left(t_{m}\right), H \overline{\mathbf{x}}_{1}\left(t_{m}+1\right), \ldots, H \overline{\mathbf{x}}_{1}\left(t_{m}+k-1\right)\right) .
\end{gathered}
$$

Remark 8: It is worthwhile noticing that the same reasoning adopted at the end of the previous section for observability applies to reconstructibility. This ensures that, independently of $\mathbf{u}(t), t \in \mathbb{Z}_{+}$, if the $\mathrm{BCN}$ is reconstructible, the final state can be identified within $\frac{N(N-1)}{2}$ steps.

Example 4: Consider a BCN (11) and suppose that

$$
\begin{aligned}
& L_{1}:=L \ltimes \delta_{2}^{1}=\left[\begin{array}{llll}
0 & 0 & 0 & 0 \\
0 & 0 & 0 & 0 \\
1 & 1 & 1 & 0 \\
0 & 0 & 0 & 1
\end{array}\right]=\left[\begin{array}{llll}
\delta_{4}^{3} & \delta_{4}^{3} & \delta_{4}^{3} & \delta_{4}^{4}
\end{array}\right], \\
& L_{2}:=L \ltimes \delta_{2}^{2}=\left[\begin{array}{llll}
0 & 0 & 0 & 0 \\
0 & 0 & 0 & 0 \\
0 & 0 & 1 & 0 \\
1 & 1 & 0 & 1
\end{array}\right]=\left[\begin{array}{llll}
\delta_{4}^{4} & \delta_{4}^{4} & \delta_{4}^{3} & \delta_{4}^{4}
\end{array}\right], \\
& H=\left[\begin{array}{llll}
1 & 1 & 0 & 1 \\
0 & 0 & 1 & 0
\end{array}\right]=\left[\begin{array}{llll}
\delta_{2}^{1} & \delta_{2}^{1} & \delta_{2}^{2} & \delta_{2}^{1}
\end{array}\right] .
\end{aligned}
$$

The $\mathrm{BCN}$ can be represented as in the following digraph:

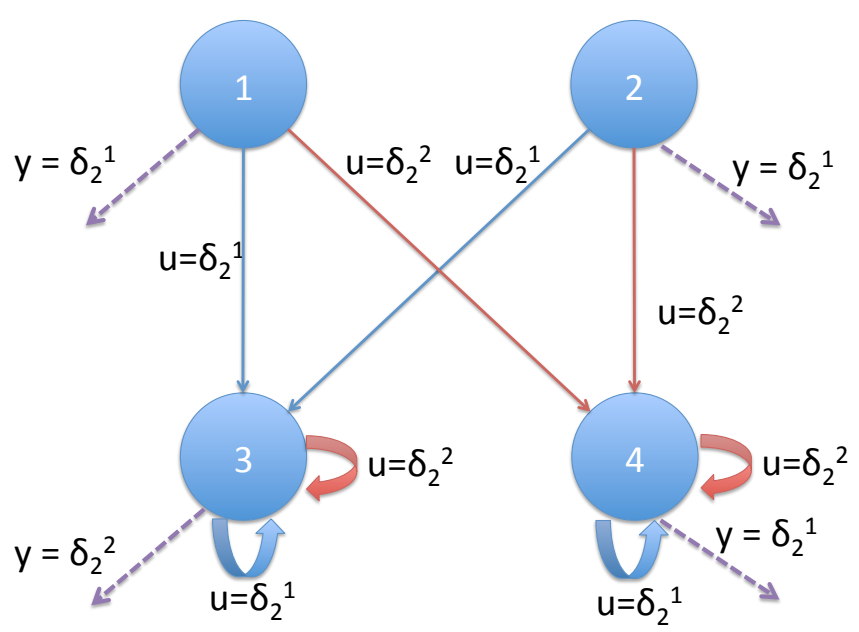

FIG. 4. Digraph corresponding to the BCN of Example 4.

It is a matter of simple computation to verify that both conditions of Theorem 4 are verified. More easily, it is immediate to verify that, independently of the input and of the initial condition, the output evolution from $T=1$ onward is either $\delta_{2}^{1}$ or $\delta_{2}^{2}$. In the former case it means that $\mathbf{x}(T)=\mathbf{x}(t)=\delta_{4}^{4}$ for every $t \geq T$, and in the latter case we have $\mathbf{x}(T)=\mathbf{x}(t)=\delta_{4}^{3}$ for every $t \geq T$. Consequently, the $\mathrm{BCN}$ is reconstructible.

\section{STATE OBSERVERS}

The aim of this section is that of designing a state observer for a $\mathrm{BCN}$, by this meaning a Boolean system that receives, as its inputs, the BCN input and output, and produces as its output an estimate of the current $\mathrm{BCN}$ state. We propose two different solutions. Both of them can be described by means of Boolean control networks. However, they will be derived as linear Boolean systems whose describing vectors consist of blocks of canonical vectors, and hence we will introduce them by making use of this more intuitive set-up. Clearly, a necessary condition for the existence of a state observer is that the $\mathrm{BCN}$ is reconstructible in some interval $[0, T]$.

\section{A. Shift-register observer}

We first notice that, due to the time-invariance of the $\mathrm{BCN}$, reconstructibility implies that the simultaneous knowledge of the input and the output trajectories, $\mathbf{u}(\tau)$ and $\mathbf{y}(\tau), \tau \in[t-$ $T, t]$, allows ${ }^{7}$ to uniquely determine $\mathbf{x}(t)$. This ensures that the vector

$$
\mathfrak{z}(t):=\left[\begin{array}{c}
\mathbf{u}(t-T) \\
\frac{\mathbf{y}(t-T)}{\vdots} \\
\frac{\mathbf{u}(t)}{\mathbf{y}(t)}
\end{array}\right] \in \underbrace{\left(\mathcal{L}_{M} \times \mathcal{L}_{P}\right) \times \ldots \times\left(\mathcal{L}_{M} \times \mathcal{L}_{P}\right)}_{T+1},
$$

corresponding to an input/output trajectory, uniquely identifies the final state $\mathbf{x}(t)$ of the BCN. We let $\mathcal{R}$ denote the reconstructibility map that associates every such "admissible"8 vector $\mathfrak{z}(t)$ to the final state $\mathbf{x}(t) \in \mathcal{L}_{N}$. We want to implement the map $\mathcal{R}$ by means of a linear Boolean system. To this end, set $Z:=M^{T+1} P^{T+1}$ and introduce the (bijective) map $\psi:\left(\mathcal{L}_{M} \times \mathcal{L}_{P}\right) \times \ldots \times\left(\mathcal{L}_{M} \times \mathcal{L}_{P}\right) \rightarrow \mathcal{L}_{Z}$, mapping each vector $\mathfrak{z}(t)$ into the corresponding canonical vector

$$
\mathbf{z}(t):=\mathbf{u}(t-T) \ltimes \mathbf{y}(t-T) \ltimes \ldots \ltimes \mathbf{u}(t) \ltimes \mathbf{y}(t) \in \mathcal{L}_{Z} .
$$

Clearly, the set of admissible vectors $\mathfrak{z}(t)$ biuniquely corresponds to the subset of "admissible" canonical vectors in $\mathcal{L}_{Z}$. The static map

$\hat{H}: \mathcal{L}_{Z} \rightarrow \mathcal{L}_{N}: \delta_{Z}^{j} \mapsto \begin{cases}\mathcal{R}\left(\psi^{-1}\left(\delta_{Z}^{j}\right)\right), & \text { if } \delta_{Z}^{j} \text { is admissible; } \\ \delta_{N}^{1}, & \text { otherwise; }\end{cases}$ associates every admissible vector $\delta_{Z}^{j}$ in $\mathcal{L}_{Z}$ with the unique state that is reconstructible at time $t$ from $\mathfrak{z}(t)=\psi^{-1}\left(\delta_{Z}^{j}\right)$. If $\delta_{Z}^{j}$ is not admissible, $\hat{H}$ associates it with an arbitrary state in

\footnotetext{
${ }^{7}$ It is worthwhile to remark that even when $T$ is the smallest time instant such that reconstructibility in $[0, T]$ is possible, nonetheless, for sufficiently large values of $t$, the minimum value of $\tilde{T}$ such that from $\mathbf{u}(\tau)$ and $\mathbf{y}(\tau), \tau \in$ $[t-\tilde{T}, t]$, one can determine $\mathbf{x}(t)$ can be significantly smaller than $T$.

${ }^{8}$ In the following, an input/output trajectory $\left\{\left[\begin{array}{c}\mathbf{u}(t) \\ \mathbf{y}(t)\end{array}\right]: t \in \mathbb{Z}_{+}\right\}$will be called "admissible" if there exists an initial state $\mathbf{x}(0)$ such that when the input sequence at time $t$ is $\mathbf{u}(t)$, then the corresponding output is $\mathbf{y}(t)$. Accordingly, the vector $\mathfrak{z}(t)$ in (16) will be called "admissible" if it coincides with the restriction of an admissible trajectory to the time window $[t-T, t]$.
} 
$\mathcal{L}_{Z}$ (e.g. $\left.\delta_{N}^{1}\right)$. Consequently, the following Boolean system ${ }^{9}$ and

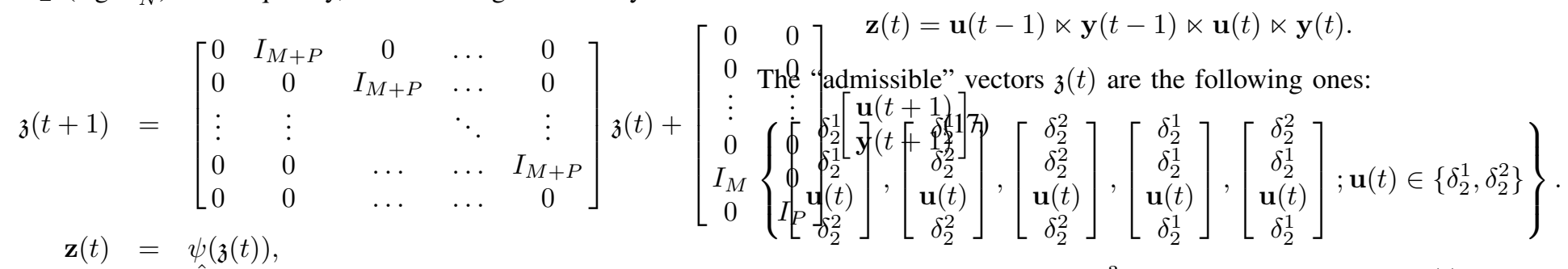

$\hat{\mathbf{x}}(t)=\hat{H} \mathbf{z}(t)$,

$\mathcal{R}$ is the map that salssociates $\delta_{4}^{3}$ to all admissible vectors $\mathfrak{z}(t)$

once initialized with an arbitrary $\mathfrak{z}(0) \in\left(\mathcal{L}_{M} \times \mathcal{L}_{P}\right) \times \ldots \times$ $\left(\mathcal{L}_{M} \times \mathcal{L}_{P}\right)$, produces an estimate $\hat{\mathbf{x}}(t)$ of the $\mathrm{BCN}$ state satisfying

$$
\hat{\mathbf{x}}(t)=\mathbf{x}(t), \quad \forall t \geq T .
$$

The previous observer may be described as a modified version of a BCN (11), namely as

$$
\begin{aligned}
\mathbf{z}(t+1) & =M \ltimes \mathbf{u}(t+1) \ltimes \mathbf{y}(t+1) \ltimes \mathbf{z}(t), \\
\hat{\mathbf{x}}(t) & =\hat{H} \mathbf{z}(t),
\end{aligned}
$$

for a suitable choice of the matrix ${ }^{10} M \in \mathcal{L}_{Z \times M P Z}$. The substitution of $\mathbf{u}$ with the pair $(\mathbf{u}, \mathbf{y})$ is rather natural, since this pair represents the observer input. However, differently from a standard BCN (11), the values of the pair we need to update the state vector at $t+1$ is not $(\mathbf{u}(t), \mathbf{y}(t))$, but $(\mathbf{u}(t+1), \mathbf{y}(t+1))$. This is unavoidable, since the state estimate at time $t$ naturally requires the information on the output at the same time instant.

Clearly, the proposed solution finds an immediate adaptation to the case of BNs. In that case, it is sufficient to replace the pair $(\mathbf{u}, \mathbf{y})$ with the output $\mathbf{y}$ alone, thus getting a state observer that takes the following form

$$
\begin{aligned}
\mathbf{z}(t+1) & =M \ltimes \mathbf{y}(t+1) \ltimes \mathbf{z}(t), \\
\hat{\mathbf{x}}(t) & =\hat{H} \mathbf{z}(t),
\end{aligned}
$$

with

$\mathbf{z}(t):=\mathbf{y}(t-T) \ltimes \mathbf{y}(t-T+1) \ltimes \ldots \ltimes \mathbf{y}(t) \in \mathcal{L}_{Z}, \quad Z:=P^{T+1}$.

We illustrate the previous method by means of a simple example.

Example 5: Consider the reconstructible $\mathrm{BCN}$ of Example 4. In this case $T=1$,

$$
\mathfrak{z}(t)=\left[\begin{array}{c}
\mathbf{u}(t-1) \\
\mathbf{y}(t-1) \\
\mathbf{u}(t) \\
\mathbf{y}(t)
\end{array}\right],
$$

\footnotetext{
${ }^{9}$ The symbol + in the following equation denotes the Boolean sum, but one may notice that, since the nonzero entries in the two vectors are necessarily located in different positions, this can be seen also as a standard sum.

${ }^{10}$ If we introduce the block matrix (also called "dummy operator" in [8]) $\Phi_{k, h}:=\underbrace{\left[\begin{array}{llll}I_{k} & I_{k} & \ldots & I_{k}\end{array}\right]}_{h \text { times }}$, then it is not hard to prove that

$$
M=\Phi_{M^{T} P^{T, P}} \ltimes \Phi_{M^{T} P^{T+1}, M} \ltimes W,
$$
}

where $W$ is the product of suitable swap matrices (see [8], page 63), that perform the variable permutation $W \ltimes \mathbf{u}(t+1) \ltimes \mathbf{y}(t+1) \ltimes \mathbf{z}(t)=\mathbf{z}(t) \ltimes$ $\mathbf{u}(t+1) \ltimes \mathbf{y}(t+1)$. whose last block is $\delta_{2}^{2}$, and $\delta_{4}^{4}$ to all admissible vectors $\mathfrak{z}(t)$ whose last block is $\delta_{2}^{1}$. The admissible vectors $\mathbf{z}(t)$ are

$$
\left\{\delta_{16}^{1}, \delta_{16}^{2}, \delta_{16}^{3}, \delta_{16}^{4}, \delta_{16}^{6}, \delta_{16}^{8}, \delta_{16}^{9}, \delta_{16}^{11}, \delta_{16}^{14}, \delta_{16}^{16}\right\} .
$$

While $\delta_{16}^{1}, \delta_{16}^{3}, \delta_{16}^{9}$, and $\delta_{16}^{11}$ correspond to the final state $\hat{\mathbf{x}}(t)=$ $\delta_{4}^{4}$, all the other admissible vectors correspond to $\hat{\mathbf{x}}(t)=\delta_{4}^{3}$. This implies that the matrix $\hat{H}$ takes the following form:

$\hat{H}=\left[\begin{array}{llllllllllllllllllllllll}\delta_{4}^{4} & \delta_{4}^{3} & \delta_{4}^{4} & \delta_{4}^{3} & * & \delta_{4}^{3} & * & \delta_{4}^{3} & \delta_{4}^{4} & * & \delta_{4}^{4} & * & * & \delta_{4}^{3} & * & \delta_{4}^{3}\end{array}\right.$ where the symbol $*$ denotes an arbitrary canonical vector in $\mathcal{L}_{4}$ (for instance $\delta_{4}^{1}$ ).

\section{B. Multiple states observers}

We now propose an alternative solution for the state reconstruction of the $\mathrm{BCN}$ (11). To this end, we consider the set $\mathcal{C}_{\sim, 1}$ of all the indistinguishability classes in 1 step, namely all classes of state vectors that produce the same output value: $\left\{i \in[1, N]: H \delta_{N}^{i}=\delta_{P}^{h}\right\}, h \in[1, P]$. We denote by $\nu$ the largest of the cardinalities of such classes

$$
\nu:=\max _{h \in[1, P]}\left|\left\{i \in[1, N]: H \delta_{N}^{i}=\delta_{P}^{h}\right\}\right| .
$$

The state of the observer is a vector $\mathbf{z}(t) \in \underbrace{\mathcal{L}_{N} \times \ldots \times \mathcal{L}_{N}}_{\nu \text { times }}$ with $\nu$ blocks, denoted by $\mathbf{z}_{i}(t), i \in[1, \nu]$, each of them belonging to $\mathcal{L}_{N}$.

We first describe the algorithm, whose purpose is that of updating the (multiple, i.e. $\nu$ ) estimates of the $\mathrm{BCN}$ state at time $t+1$ (the $\nu$ blocks of $\mathbf{z}(t+1)$ ), based on the estimates we had at time $t$ (the $\nu$ blocks of $\mathbf{z}(t)$ ) and on the current input sample $\mathbf{u}(t)$. At each time $t$, the $i$ th state estimate $\mathbf{z}_{i}(t)$ is consistent with a number of input and output samples not smaller than those any estimate $\mathbf{z}_{j}(t)$, with $j>i$, is compatible with. We will further comment on the rationale underlying the algorithm after having described it. We denote by arr and dep the indices of the current block of the "arrival state" $\mathbf{z}(t+1)$ and of the current block of the "departure state" $\mathbf{z}(t)$, respectively, considered by the algorithm. Once we have considered all $\nu$ blocks of $\mathbf{z}(t)$, dep takes values greater than $\nu$. On the other hand, when we have updated all the $\nu$ blocks of $\mathbf{z}(t+1)$ the algorithm iteration is completed, and the time variable is updated. Also, we introduce two sets: $D_{t}$ and $A_{t+1}$. We initialize the set $D_{t}$ with all the indices of the states that are compatible with the output sample $\mathbf{y}(t)$, while $A_{t+1}$ is initially empty. As the algorithm proceeds, all these indices are moved from $D_{t}$ to $A_{t+1}$. 
In detail, the observer state updates according to the following algorithm:

Inizialization: Set $t:=0$, arr $:=1, \operatorname{dep}:=1, D_{t}:=\{i \in[1, N]$ : $\left.H \delta_{N}^{i}=\mathbf{y}(t)\right\}$ and $A_{t+1}:=\emptyset$.

Step 1: Until $\operatorname{arr} \leq \nu, \operatorname{dep} \leq \nu$ and $D_{t} \neq \emptyset$,

if $\mathbf{z}_{\text {dep }}(t)=\delta_{N}^{j}$ with $j \in D_{t}$,

then

$\mathbf{z}_{\text {arr }}(t+1)=L \ltimes \mathbf{u}(t) \ltimes \mathbf{z}_{d e p}(t)$

$\operatorname{arr}:=\operatorname{arr}+1$

dep $:=\operatorname{dep}+1$

$D_{t}:=D_{t} \backslash\{j\}$;

$A_{t+1}:=A_{t+1} \cup\{j\}$;

otherwise

$\operatorname{dep}:=\operatorname{dep}+1$.

Step 2: $\quad$ If $\operatorname{arr} \leq \nu$ and $D_{t} \neq \emptyset$ then

set $j:=\min \left\{i \in[1, N]: i \in D_{t}\right\}$;

$\mathbf{z}_{\text {arr }}(t+1)=L \ltimes \mathbf{u}(t) \ltimes \delta_{N}^{j} ;$

$\operatorname{arr}:=\operatorname{arr}+1$

$\operatorname{dep}:=\operatorname{dep}+1$

$D_{t}:=D_{t} \backslash\{j\}$;

$A_{t+1}:=A_{t+1} \cup\{j\}$;

go back to Step 2;

otherwise

go to Step 3;

Step 3: If $\operatorname{arr} \leq \nu$ and $D_{t}=\emptyset$ then

set $j:=\min \left\{i \in[1, N]: i \in A_{t+1}\right\}$;

$\mathbf{z}_{\text {arr }}(t+1)=L \ltimes \mathbf{u}(t) \ltimes \delta_{N}^{j} ;$

$\operatorname{arr}:=\operatorname{arr}+1$

$\operatorname{dep}:=\operatorname{dep}+1$

go back to Step 3;

otherwise

go to Step 4;

Step 4: Set $t:=t+1$, arr $:=1$, dep $:=1, D_{t}:=\{i \in[1, N]$ : $\left.H \delta_{N}^{i}=\mathbf{y}(t)\right\}$ and $A_{t+1}:=\emptyset$, and

go back to Step 1 .

The idea underlying the algorithm is

(a) first, all distinct blocks $\mathbf{z}_{d e p}(t)$ of $\mathbf{z}(t)$ are ordinately considered: if they are compatible with the output sample $\mathbf{y}(t)$ they are used to determine the current block $\mathbf{z}_{\text {arr }}(t+1)$ by means of the formula $\mathbf{z}_{a r r}(t+1)=L \ltimes \mathbf{u}(t) \ltimes \mathbf{z}_{d e p}(t)$. If not, they are simply neglected;

(b) if the number $d_{t}$ of such distinct blocks in $\mathbf{z}(t)$ is smaller than the cardinality $\nu_{t}$ of the class $\left\{i \in[1, N]: H \delta_{N}^{i}=\mathbf{y}(t)\right\}$, then $\nu_{t}-d_{t}$ blocks of the vector $\mathbf{z}(t+1)$ are evaluated by making use of the remaining $\nu_{t}-d_{t}$ canonical vectors $\delta_{N}^{j}$ of $\left\{i \in[1, N]: H \delta_{N}^{i}=\mathbf{y}(t)\right\}$, through the formula $\mathbf{z}_{\text {arr }}(t+1)=$ $L \ltimes \mathbf{u}(t) \ltimes \delta_{N}^{j} ;$

(c) if $\nu_{t}<\nu$, the remaining blocks in $\mathbf{z}(t+1)$ are evaluated by making use of $\delta_{N}^{i}$, where $i$ is the smallest index in $\{i \in$ $\left.[1, N]: H \delta_{N}^{i}=\mathbf{y}(t)\right\}$.

It is clear that the first block of $\mathbf{z}(t), t>0$, is the state that has proved to be compatible with the largest number of output and input samples. In particular, at time $T+1$ the state $\mathbf{z}_{1}(T+1)$ is compatible with the input-output trajectory $(\mathbf{u}(t), \mathbf{y}(t)), t \in[0, T]$. So, independently of the initial state of the observer, the state updating algorithm is conceived in such a way that, if the original $\mathrm{BCN}$ is reconstructible in $[0, T]$, then $\mathbf{z}_{1}(T+1)=\mathbf{x}(T+1)$.
Example 6: We illustrate also this algorithm by referring to Example 4 . We notice that $\mathcal{C}_{\sim, 1}$ consists of two classes:

$$
\begin{aligned}
& \left\{i \in[1,4]: H \delta_{4}^{i}=\delta_{2}^{1}\right\}=\{1,2,4\}, \\
& \left\{i \in[1,4]: H \delta_{4}^{i}=\delta_{2}^{2}\right\}=\{3\} .
\end{aligned}
$$

The index $\nu$ coincides with the cardinality of the largest class and hence it is 3 . Suppose that we have the input trajectory $\mathbf{u}(0)=\delta_{2}^{2}, \mathbf{u}(1)=\delta_{2}^{1}, \mathbf{u}(2)=\delta_{2}^{2}, \ldots$, and the corresponding output trajectory $\mathbf{y}(0)=\delta_{2}^{1}, \mathbf{y}(1)=\delta_{2}^{1}, \mathbf{y}(2)=\delta_{2}^{1}, \ldots . \mathrm{We}$ can initialize the observer state, for instance, with $\mathbf{z}(0)=$ $\left[\begin{array}{lll}\delta_{4}^{1} & \delta_{4}^{3} & \delta_{4}^{2}\end{array}\right]^{\top}$.

We set $t:=0, D_{0}:=\{1,2,4\}$ and $A_{1}:=\emptyset$. By applying the previous algorithm, we easily find

$$
\begin{aligned}
& \mathbf{z}_{1}(1):=L \ltimes \mathbf{u}(0) \ltimes \mathbf{z}_{1}(0)=\delta_{4}^{4}, \\
& \mathbf{z}_{2}(1):=L \ltimes \mathbf{u}(0) \ltimes \mathbf{z}_{3}(0)=\delta_{4}^{4}, \\
& \mathbf{z}_{3}(1):=L \ltimes \mathbf{u}(0) \ltimes \delta_{4}^{4}=\delta_{4}^{4} .
\end{aligned}
$$

At $t=1$, we have $D_{1}:=\{1,2,4\}$ and $A_{2}:=\emptyset$, and the algorithm gives

$$
\begin{aligned}
& \mathbf{z}_{1}(2):=L \ltimes \mathbf{u}(1) \ltimes \mathbf{z}_{1}(1)=\delta_{4}^{4}, \\
& \mathbf{z}_{2}(2):=L \ltimes \mathbf{u}(1) \ltimes \delta_{4}^{1}=\delta_{4}^{4}, \\
& \mathbf{z}_{3}(2):=L \ltimes \mathbf{u}(1) \ltimes \delta_{4}^{2}=\delta_{4}^{4} .
\end{aligned}
$$

Clearly, $\mathbf{z}_{1}(2)=\delta_{4}^{4}=\mathbf{x}(2)$.

Also, in this case, the state observer could be described by means of a BCN, upon replacing each vector $\mathbf{z}(t)$ with the corresponding "canonical" representation $\xi(t)$, thus getting a system of the following kind

$$
\begin{aligned}
\xi(t+1) & =M \ltimes \mathbf{u}(t) \ltimes \mathbf{y}(t) \ltimes \xi(t), \\
\hat{\mathbf{x}}(t) & =\hat{H} \xi(t),
\end{aligned}
$$

for suitable matrices ${ }^{11} M$ and $\hat{H}$.

To conclude the section, we want to compare the different philosophies underlying the two proposed observers. The shiftregister observer is essentially the dynamic implementation of the static map $\mathcal{R}$. Under the reconstructibility assumption, the map $\mathcal{R}$ allows to reconstruct the state of the BCN at time $t$ from the input and output values in the window $[t-T, t]$. Such input and output samples, ordered from the oldest to the most recent, constitute the blocks of the observer state at time $t$, whose updating consists in removing the oldest samples and acquiring the new ones. The key ingredient of the $\mathrm{BCN}$ state

\footnotetext{
${ }^{11}$ It is difficult to find a general expression for the matrix $M$, whose existence is however ensured by the fact that every triple $(\mathbf{u}(t), \mathbf{y}(t), \mathbf{z}(t))$ is mapped into a single vector $\mathbf{z}(t+1)$ and, consequently, each canonical vector $\mathbf{u}(t) \ltimes \mathbf{y}(t) \ltimes \xi(t)$ is mapped into a unique canonical vector $\xi(t+1)$. On the other hand, the matrix $\hat{H}$ can be easily determined along the lines we used for the previous observer. Indeed, as $\hat{\mathbf{x}}(t)=\mathbf{z}_{1}(t)$, we can first determine the swap matrix $W$ such that
}

$W \ltimes \xi(t)=W \ltimes \mathbf{z}_{1}(t) \ltimes \mathbf{z}_{2}(t) \ltimes \ldots \ltimes \mathbf{z}_{\nu}(t)=\mathbf{z}_{2}(t) \ltimes \ldots \ltimes \mathbf{z}_{\nu}(t) \ltimes \mathbf{z}_{1}(t)$, and then apply a finite sequence of dummy operators, to delete the variables $\mathbf{z}_{2}(t) \ltimes \ldots \ltimes \mathbf{z}_{\nu}(t)$. This implies that

$$
\hat{H}=\Phi_{N, N} \ltimes \Phi_{N^{2}, N} \ltimes \ldots \ltimes \phi_{N^{\nu-1}, N} \ltimes W .
$$


estimation is represented by the static map $\hat{H}$, that acts on the "canonical representation" $\mathbf{z}(t)$ of the observer state and realizes the map $\mathcal{R}$.

On the other hand, the multiple states observer proceeds according to a different rationale. It memorizes all the current state estimates, and it updates only those that are compatible with the current output sample, by making use of the system laws and the knowledge of the current input value. So, the observer state consists of $\nu$ possible estimates of the $\mathrm{BCN}$ state. The blocks with lower index in the observer state are those that have proved to be compatible with a larger number of input/output samples, and for this reason once they prove to be compatible with the input and output for $T+1$ consecutive time instants, they provide the real value of the $\mathrm{BCN}$ state. It is worth noticing that the key ingredient of this observer is the state updating law, while the static output map, that provides the BCN state estimate, simply consists in selecting the first block of the observer state vector.

\section{REFERENCES}

[1] T. Akutsu, M. Hayashida, W.-K. Ching, and M.K. Ng. Control of Boolean networks: hardness results and algorithms for tree structured networks. J. Theoret. Biol., 244:670-679, 2007.

[2] R.A. Brualdi and H.J. Ryser. Combinatorial matrix theory. Cambridge Univ.Press, Cambridge (GB), 1991.

[3] D. Cheng. Input-state approach to Boolean Networks. IEEE Trans. Neural Networks, 20, (3):512 - 521, 2009.

[4] D. Cheng. Disturbance decoupling of Boolean control networks. IEEE Trans. Automatic Control, 56:2-10, 2011.

[5] D. Cheng, Q. Hongsheng, and Y. Zhao. Synthesis of Boolean networks via semi-tensor product. In Proceedings of the 30th Chinese Control Conference (CCC2011), pages 6-17, Yantai, China, 2011.

[6] D. Cheng and H. Qi. Linear representation of dynamics of Boolean Networks. IEEE Trans. Automatic Control, 55, (10):2251 - 2258, 2010.

[7] D. Cheng and H. Qi. State-space analysis of Boolean Networks. IEEE Trans. Neural Networks, 21, (4):584 - 594, 2010.

[8] D. Cheng, H. Qi, and Z. Li. Analysis and control of Boolean networks. Springer-Verlag, London, 2011.

[9] Li Fangfei, Sun Jitao, and Wu Qi-Di. Observability of Boolean Control Networks with state time delays. IEEE Trans. Neural Networks, 22, (6): $948-954,2011$.

[10] L. Farina and S. Rinaldi. Positive linear systems: theory and applications. Wiley-Interscience, Series on Pure and Applied Mathematics, New York, 2000.

[11] E. Fornasini and M.E. Valcher. Observability and reconstructibility of Boolean control networks. In Proceedings of the 51st Conference on Decision and Control (CDC2012), page to appear, Maui, Hawaii, 2012.

[12] D.G. Green, T.G. Leishman, and S. Sadedin. The emergence of social consensus in Boolean networks. In Proceedings of the IEEE Symp. Artificial Life (ALIFE07), page 402408, Honolulu, HI, 2007.

[13] R.E. Kalman, P.L. Falb, and M.A. Arbib. Topics in mathematical systems theory. McGraw Hill, 1969.

[14] S.A. Kauffman. Metabolic stability and epigenesis in randomly constructed genetic nets. J. Theoretical Biology, 22:437467, 1969.

[15] K.H. Kim. Boolean matrix theory and applications. M. Dekker Inc., NY, 1982.

[16] D. Laschov and M. Margaliot. A maximum principle for single-input Boolean Control Networks. IEEE Trans. Automatic Control, 56, no. 4:913-917, 2011.

[17] D. Laschov and M. Margaliot. A new approach to the controllability of Boolean Control Networks. submitted, 2011.

[18] D. Laschov and M. Margaliot. Controllability of Boolean control networks via the Perron-Frobenius theory. Automatica, 2012.

[19] H. Li and Y. Wang. Boolean derivative calculation with application to fault detection of combinational circuits via the semi-tensor product method. Automatica, 48, (4):688-693, 2012.

[20] H. Li, Y. Wang, and Z. Liu. Simultaneous stabilization of Boolean control networks via semi-tensor product method. In Proceedings of the 30th Chinese Control Conference (CCC2011), pages 6386 - 6391, Yantai, China, 2011.
[21] Y. Lou and Y. Hong. Multi-agent decision in Boolean networks with private information and switching interconnection. In Proceedings of the 29th Chinese Control Conference (CCC 2010), pages 4530 - 4535, Beijing, China, 2010.

[22] D.G. Luenberger. Introduction to dynamical systems. J.Wiley \& Sons Inc., 1979.

[23] I. Shmulevich, E.R. Dougherty, S. Kim, and W. Zhang. From Boolean to probabilistic Boolean networks as models of genetic regulatory networks. Proc. IEEE, 90, no. 11:1778-1792, 2002. 